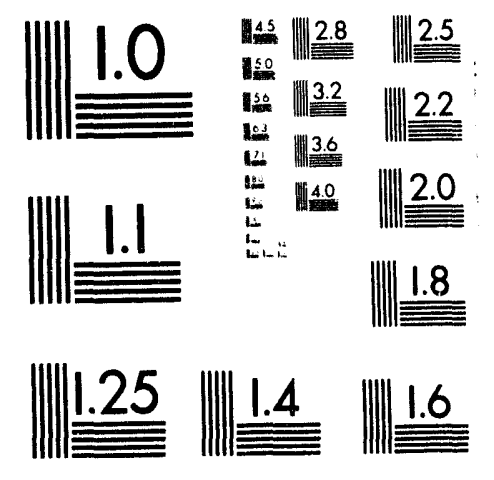



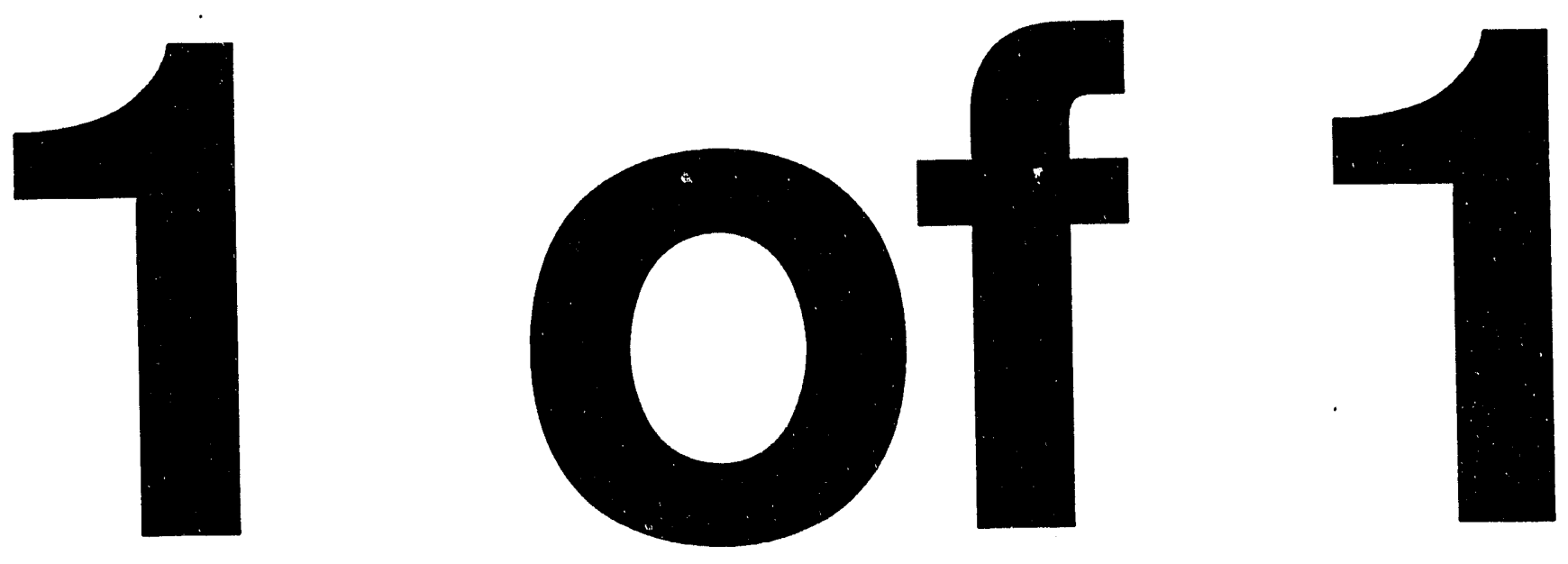


\begin{abstract}
This report was prepared as an account of work sponsored by an agency of the United States Government. Neither the United States Government nor any agency thereof, nor any of their employees, makes any warranty, express or implied, or assumes any legal liability or responsibility for the accuracy, completeness, or usefulness of any information, apparatus, product, or process disclosed, or represents that its use would not infringe privately owned rights. Reference herein to any specific commercial product, process, or service by trade name, trademark, manufacturer, or otherwise does not necessarily constitute or imply its endorsement, recommendation, or favoring by the United States Government or any agency thereof. The views and opinions of authors expressed herein do not necessarily state or reflect those of the United States Government or any agency thereof.
\end{abstract}

\title{
QUANTUM DYNAMICS IN DUAL SPACES
}

\author{
E.C.G. Sudarshan \\ Center for Particle Physics, Department of Physics \\ University of Texas, Austin, TX 78712
}

Quntum mechanics gives us information about spectra of dynamical variables and transition rates including scattering cross sections. They can be exhibited as spectral information in analytically continued spaces and their duals. Quantum mechanics formulated in these generalized spaces is used to study scattering and time evolution. It is shown that the usual asymptotic condition is inadequate to deal with scattering of composite or unstable particles. Scattering theory needs amendment when the interacting system is not isospectral with the free Hamiltonian, and the amendment is formulated. Perturbation theory in generalized spaces is developed and used to study the deletion and augmentation of the spectrum of the Hamiltonian. A complete set of algebraically independent constants for an interacting system is obtained. The question of the breaking of time symmetry is discussed.

\section{Introduction}

Quantum mechanical systems are usually defined by an algebra of operators over the field of complex numbers acting as linear operators on a vector space of states with a defined inner product [1]. The vector space is usually taken to be a separable Hilbert space [2]. In a series of papers during the seventies it has been shown that the Hilbert space structure can be generalized in two directions. One: to consider pairs of dual vector spaces which are analytic continuations of dense sets of analytic vectors which are realizations of the Hilbert space. This leads to generalized quantum mechanics [3]. Two: Nonnormalized vectors and a wider class of functions realizing vectors can be introduced with a suitable set of dual vectors [4]; when this is done in the context of eigenvectors belonging to the continuum one gets the rigged Hilbert space [5]; 


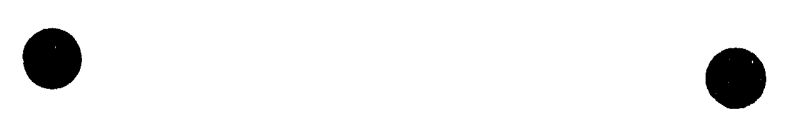

but the formalism of dual spaces is more general and makes it unnecessary to start with Hilbert spaces and selfadjoint operators acting on this space [6]. In this paper we continue our study of generalized quantum mechanics in terms of dual spaces.

Much of the physical interpretation of quantum theories include expectation values and transition probabilities of chosen dynamical variables with a preassigned basis. But the generic properties of the system like spectra of operators are identified with physically significant properties of the system. In previous studies it has been shown that the notion of the spectrum of a (self adjoint) dynamical operator in generalized quantum mechanics is not unique and does depend on the context in which it is evaluated. Rather than consider this as an inconvenience we may view this as an opportunity to obtain resonance frequencies and widths as spectral information. This is, in a sense, the realization of the Heisenberg programme for atomic physics in which spectral intensities, frequencies and polarizations should enter directly into the quantum theory of the atom. We may attempt to realize transition rates as spectral information in generalized quantum mechanics. This goal has been partially achieved in earlier papers but in this paper a more comprehensive program is implemented.

The first systematic treatment of analytically continued spectra was undertaken in the theory of Master Analytic Representations of noncompact groups [7]. Inspired by the "unitary trick" of H. Weyl and the complex rank tensors ("extensors") of PAM Dirac and "exspinors" of HarishChandra [8], in the theory of Master Analytic Representations one uses shift operators $\left(E_{\alpha}\right.$ in the sense of E. Cartan) which increase the continuous eigenvalues of self adjoint operators by complex values; these are then interpreted as linear functionals on analytic functions. This method was applied to finding all irreducible representations of a variety of noncompact groups obtaining all the irreducible representations from the specialization of a generic "master" analytic representation [9].

The analytically continued spectra came up in the formulation of solvable models in the context of unstable particles [3]. Analytically continuing the complete set of (ideal) states for 
the Dirac-Friedrichs-Lee model we have shown how families of generalized dual vector pairs can be constructed and with full mathematical rigor $[4,10]$.

These considerations can be extended from the vector space treatment to the density matrix treatment, especially in the context of time evolution. The Hamiltonian evolution is replaced by the Liouvillian evolution. Despite the lack of semiboundedness of the Hamiltonian, the Liouvillian evolution can also be analytically continued. On analytic continuation we encounter the metastable excitations of the density matrix and the decaying components of the density matrix $[3,11]$.

Many of the questions of scattering and decay that arise are not the intrinsic property of one Hamiltonian but the properties appropriate to a particular realization of that Hamiltonian operator. In the case of scattering it has long been emphasized that we have to consider two Hamiltonians, the true "total Hamiltonian" and an isospectral "comparison Hamiltonian" [12]. While sometimes the comparison Hamiltonian is identical with a "free Hamiltonian" this is neither necessary nor desirable. The free Hamiltonian is generally not isospectral with the total Hamiltonian. The specific requirement of being isospectral (with the proper multiplicity!) enables us to get a unitary intertwining between the total Hamiltonian and the comparison Hamiltonian, while the free Hamiltonian people get only isometry rather than unitarity for the intertwining Möller operator. There are also other problems with wave function normalization that are encountered by people who rely on asymptotic conditions and free Hamiltonians. This notwithstanding, scattering is a property of the relationship between two Hamiltonians.

The use of the generalized spectral decomposition is useful in other areas of physics including optics and dynamical maps. In the applications to maps it becomes quite clear that dual pairs of vector spaces are the appropriate framework for dealing with generalized spectra.

In Sec. 2 we formulate analytic Hamiltonian systems and demonstrate the generalized spectra in the dual spaces. For the state vectors we compute the survival amplitude and demonstrate that they may be computed in any pair of the dual spaces. The discrete and continuum states at 
complex energies are demonstrated and the resolutions of the identity (completeness of states) is demonstrated for each pair of dual spaces. The case of potential scattering is an important special case. The forward and backward time evolutions are compared and the appropriateness of specific pairs of dual spaces breaking the time symmetry is pointed out. The corresponding development of the density matrices is also explored.

In Sec. 3 we explore scattering states with a total Hamiltonian separated into a free Hamiltonian and an interaction. The general solution is given and the problems that arise when the free Hamiltonian and the total Hamiltonian are not isospectral are pointed out. When bound states enter scattering states as a constituent further complications do arise. The method of resolving these complications is formulated. The time evolution of the state vector and of the density matrix is examined in detail. A complete set of pseudo densities constitute a basis for densities. The extremely delicate operations needed for defining density matrices for energies in the continuum make it very necessary to exercise great care in computing the time development. The evolution is still unitary in the Hilbert space but contains metastable discrete pseudo densities for the generalized dual spaces. The survival probabilities are obtained directly. The approximations in which the evolutions appear irreversible are briefly sketched. Despite the reversibility of the time evolution we can see that exponentially damped evolution in the absolute value of time obtain by virtue of the appropriate selection of the generalized dual spaces. This choice breaks time symmetry.

In Sec. 4 the decomposition of the physical state into the direct sum of two analytic vectors in a wider space of generalized states is shown as a further illustration of the breaking of time symmetry by redefinition of the "states". The special time dependencies of the two generalized pseudo state vectors and their relationship to the Beit-Wigner approximation [20] and to the Gamow vectors [21] is bought out. A model of a decaying system in terms of an exponentially weighted integral of these pseudovectors is shown to furnish a realization of the semigroup of time evolutions. In Sec. 5 the corresponding decomposition of a density matrix into pseudo density 
matrices in a larger space is discussed and its relation to the Hilbert transform is demonstrated.

Returning to scattering systems in which the total Hamiltonian is isospectral to the free Hamiltonian, we define in Sec. 6 the complete set of invariants for a system with $N$ degrees of freedom and exhibit $2 N-1$ algebraically independent constants including the total Hamiltonian [12]; and that $N$ of them are a complete commuting set. Special mention is also made of systems with multiply degenerate spectrum. In Sec. 7 we exhibit the analytic continuation of the constants of motion. The evaluation of the expectation value of these constants of motion is carried out and they are shown to furnish $2 N-2$ constants of motion in addition to the expectation value of the energy. The calculations necessarily involve the matrix elements in a complete basis of generalized vectors or pseudo densities. The singularities of these matrix elements as distributions and as analytic functions are also explored, along with the matrix elements of several simple operators.

In Sec. 8 we study the $S$-matrix and the scattering amplitude in the Hilbert space and in the generalized dual pairs of spaces. It is shown to be unitary and defined over all states of the interacting system with the correct multiplicity. The question of scattering in the cases when some of the states contain composite particles is taken up and the necessary renormalizations of the Möller operator worked out. The corresponding calculations for the density matrix are also exhibited and shown to yield directly observable quantities. The optical theorem is reproduced in these density matrix calculations. The analytically continued spaces may be equally used for calculating these quantities.

In Sec. 9 we develop perturbation theory in the generalized spaces [13]. The change in the spectrum on interaction is explicity brought out. Both che (off-shell) scattering amplitude and the wave functions are computed; the time dependence of the wavefunction is recovered in perturbation theory. The possible incompatibility between the analytic properties of the scattering amplitude and the spectrum of states both in terms of redundant poles [14] and in terms of physical states which do not produce singularities in the scattering amplitude is demonstrated. 
Some models with closed form solutions which illustrate these results are discussed.

Sec. 10 gives a general discussion of density functionals realizing states on an algebra of dynamical variables which go beyond the ordinary notion of density matrix [15]. It is shown how most of the results discussed above ca:l be adapted to this perspective and how they "soften" many of the singularities. Scattering ross sections are evaluated in this context. The time asymmetry of these considerations when a cross-section appears [16] is pointed out and traced to the asymmetric treatment of the energy diagonal initial scattering configuration and the correlations in the final state.

\section{Generalized Dual Spaces and Analytic Hamiltonians}

Define a vector space of complex valued functions of a set of real variables $\{\lambda\}$ bounded from below over the field of complex numbers with the standard linear operations of addition and (complex) scalar multiplication. A familiar norm is the $L^{2}$ norm

$$
(\phi, \phi)=\int_{\lambda_{0}}^{\lambda_{1}} \phi^{*}(\{x\}) \phi(\{x\}) d\{x\}
$$

where, for most applications we may choose $\left\{\lambda_{0}\right\}=\{0\},\left\{\lambda_{1}\right\}=\{\infty\}$; but this is not essential. The nested projections $\Pi(\{x\})$ obeying

$$
\begin{aligned}
\Pi(\{\lambda\}) \Pi(\{\mu\}) & =\Pi(\{\mu\}) \Pi(\{\lambda\})=\Pi(\{\nu\}),\{\nu\}=\min (\{\lambda\},\{\mu\}) \\
\Pi\left(\left\{\lambda_{0}\right\}\right. & =0 ; \Pi\left(\left\{\lambda_{1}\right\}\right)=1
\end{aligned}
$$

may be defined by

$$
\begin{aligned}
(\Pi(\{\mu\}) \phi)(\{\lambda\}) & =\theta(\{\lambda\},\{\mu\}) \phi(\{\lambda\}) . \\
\theta(\{\lambda\},\{\mu\}) & =\left\{\begin{array}{ll}
1 & \{\lambda\} \geq\{\mu\} \\
0 & \text { otherwise }
\end{array},\right.
\end{aligned}
$$

with any suitable ordering relation for the sets $\{\lambda\},\{\mu\}$. We can also define the operators $\{\Lambda\}$ by

$$
(\{\Lambda\} \phi)(\{\lambda\})=\lambda \phi(\{\lambda\})
$$


Then by introducing the ideal vectors $\phi_{\lambda}$ of $\Lambda$ we can present the integral representations

$$
\begin{aligned}
\{\Lambda\} & =\int\{\lambda\} \phi_{\{\lambda\}} \phi_{\{\lambda\}}^{*} d \lambda \\
\Pi(\{\mu\}) & =\int_{\left\{\lambda_{0}\right\}}^{\{\mu\}} \phi_{\{\lambda\}} \phi_{\{\lambda\}}^{*} d\{\lambda\} .
\end{aligned}
$$

As long as $\left\{\lambda_{1}\right\}<\{\infty\}$ there is a one parameter family of "time operators" $\mathcal{T}$ conjugate to the "energy" operated $\Lambda$ corresponding to the one parameter family with

$$
\phi\left(\left\{\lambda_{1}\right\}\right) \rightarrow e^{i \beta} \phi\left(\left\{\lambda_{0}\right\}\right)
$$

of the boundary condition, so that

$$
\phi^{*}\left(\left\{\lambda_{1}\right\}\right) \phi\left(\left\{\lambda_{1}\right\}\right) \rightarrow \phi^{*}\left(\left\{\lambda_{0}\right\}\right) \phi\left(\left\{\lambda_{0}\right\}\right)
$$

But when $\left\{\lambda_{1}\right\}=\infty$ this demands that $\phi\left(\left\{\lambda_{0}\right\}\right)=0$. This space can be completed to a Hilbert space $\mathcal{H}$.

A dense subset of vectors in $\mathcal{H}$ are analytic vectors which are realized by the square-integrable boundary values of analytic functions, analytic in some domain $\mathcal{D}$ of the complex plane. We could continue these functions within the domain $\mathcal{D}$ to functions of a complex variables $\{z\}$ defined along a contour $\Gamma$ from $\left\{\lambda_{0}\right\}$ to $\left\{\lambda_{1}\right\}$ with the norm defined by

$$
\int_{\Gamma} \phi^{*}\left(\left\{z^{*}\right\}\right) \phi(\{z\}) d\{z\}
$$

This is a norm preserving map from the dense set of analytic vectors in $\mathcal{H}$ to a set of vectors in a space $\mathcal{G}$ dependent on $\Gamma$. $\mathcal{G}$ is not yet a Hilbert space since it has to be completed. In the completion there are vectors which are not continuations of vectors in $\mathcal{H}$. So the correspondence between $\mathcal{H}$ and $\mathcal{G}$ is a correspondence between dense sets. Note that both $\phi^{*}\left(\left\{z^{*}\right\}\right)=\tilde{\phi}(\{z\})$ and $\phi(\{z\})$ are vectors in the same space $\mathcal{G}$ and they are in one-to-one antilinear correspondence.

For any normalized state $\phi$ the one-dimensional projection operator to this state is given by the operator

$$
P^{\phi}=\phi \cdot \tilde{\phi}
$$


or, more explicitly by

$$
\left(P_{\phi} \psi\right)(\{z\})=\phi(\{z\}) \int \phi^{*}\left(\left\{z^{* \prime}\right\}\right) \psi\left(\{z\}^{\prime}\right) d\left\{z^{\prime}\right\}
$$

If the state is not normalized we have the scaling factor

$$
\left\{\int_{\Gamma} \phi^{*}\left(\left\{z^{* \prime \prime}\right\}\right) \phi\left(\left\{z^{\prime \prime}\right\}\right) d\left\{z^{\prime \prime}\right\}\right\}^{-1}
$$

on the right hand side.

With these results understood we can use the ket and bra notation for the vectors in $\mathcal{G}$ and their dual, but they must be distinguished from similar symbols in $\mathcal{H}$. For convenience in writing we write $z$ in place of $\{z\}$. etc. We may then write the analogues of (1), as

$$
\begin{aligned}
\langle\phi \mid \phi\rangle & =\int_{\Gamma} \tilde{\phi}(z) \phi(z) d z=\int_{\Gamma} \phi^{*}\left(z^{*}\right) \phi(z) d z \\
P_{\phi} & =|\phi\rangle\langle\phi| \cdot\{\langle\phi \mid \phi\rangle\}^{-1} \\
1 & =\int_{\Gamma}|z\rangle\langle z| d z
\end{aligned}
$$

and

$$
\begin{aligned}
\phi_{\lambda} \rightarrow|z\rangle ;|\phi\rangle & =\int_{\Gamma} \phi(z)|z\rangle d z \\
\langle\tilde{\phi}| & =\int_{\Gamma} \phi^{*}\left(z^{*}\right)\langle z| d z
\end{aligned}
$$

If in the process of analytic continuation one encounters poles or branch points the contour would get snagged by them; and new contours can be constructed which include supplementary closed contours encircling the "poles" and pairs of "branch points". At this stage the completeness identity gets modified into

$$
\mathbf{1}=\int_{\Gamma} d z|z\rangle\left\langle z\left|+\sum_{\text {poles }}\right| z_{\gamma}\right\rangle\left\langle z_{\gamma}\left|+\int_{\beta} d \zeta\right| \zeta\right\rangle\langle\zeta| .
$$

and the scalar product becomes

$$
\langle\psi \mid \phi\rangle=\int_{\Gamma} \tilde{\psi}(z) \phi(z) d z+\sum \tilde{\psi}\left(z_{\gamma}\right) \psi\left(z_{\gamma}\right)+\int_{\beta} \tilde{\psi}(\zeta) \phi(\zeta) d \zeta
$$


Despite the fact that we use the ket and bra notation it should be recalled that these vectors are in the space $\mathcal{G}$ with the contour $\Gamma$ and therefore the scalar product of such a state $|\phi\rangle$ with a state in $\mathcal{H}$ also denoted by a ket $|f\rangle$ may not be defined. In the special case that both $|\phi\rangle$ and $\langle f|$ are in the dense analytic subset we may take the vector $\langle\varphi|$ corresponding to $\langle f|$ and evaluate the product in $\mathcal{G}$; or equally well, in $\mathcal{H}$. It must be stressed that the discrete "pole" states $\left|z_{\gamma}\right\rangle$ do not belong to the dense analytic subset; nor do discrete energy states in $\mathcal{H}$, whether bound or in the continuum belong to the dense set.

The survival amplitude ${ }^{17}$ is the correlation function

$$
A(t)=\left(\psi, e^{-i H t} \psi\right) \equiv(\psi(o), \psi(t),)
$$

computed in $\mathcal{H}$. The same quantity can be computed in any of the generalized spaces $\mathcal{G}$ by

$$
A(t)=(\phi(o), \phi(t))
$$

where $\phi(t)$ and $\tilde{\phi}(o)$ are the analytic continuations of $\psi(t)$ and $\psi^{*}(o)$. Explicitly

$$
A(t)=\int \psi^{*}(\lambda) e^{-i \lambda t} \psi(\lambda) d \lambda=\int_{\Gamma} \psi^{*}\left(z^{*}\right) e^{-i z t} \psi(z) d z
$$

The survival amplitude $A(t)$ is the same but expressed by two distinct contours. In the first expression the spectrum is along the real line $R^{+}$from 0 to $\infty$ while in the second case it is complex along $\Gamma$ from 0 to $\infty$. Excepting for the end points no two points of the spectra coincide. This is a special case of the class of representations of an algebra, in this case of the abelian time translation group $T(t)$ which is realized by

$$
T\left(t^{\prime}\right) A(t)=A\left(t+t^{\prime}\right)
$$

which is reducible in two completely nonequivalent ways. The realization may be thought of as direct integrals along the spectrum:

$$
A(t)=\int_{R^{+}} e^{-i \lambda t} d \Pi(\lambda)=\int_{\Gamma} e^{-i z t} d \Pi(z)
$$


In particular, for $t=0$,

$$
\mathbf{1}=\int_{R^{+}} d \Pi(\lambda)=\int_{\Gamma} d \Pi(z)
$$

It is possible, depending on the nature of the Hamiltonian that the contour $\Gamma$ includes discrete complex points or even contours encircling new branches that may arise in the analytic continuation from $\mathcal{H}$ to $\mathcal{G}$. In such a case the completeness integrals (resolutions of the identity) and the spectrum includes these discrete points and the new branch cuts.

$$
A(t)=\int_{\Gamma} e^{-i z t} d \Pi(z)+\sum e^{-i z_{n} t} \Pi\left(z_{n}\right)+\cdots .
$$

The Cascade model [12] which can be solved in closed form exhibits these features under suitable conditions.

A specially interesting case is obtained for the simple but important case of potential scattering where we have

$$
\begin{aligned}
H & =H_{0}+V \\
H_{0} & =-\frac{\nabla^{2}}{2 m}=-\frac{1}{2 m}\left(\frac{\partial^{2}}{\partial r^{2}}+\frac{2}{r} \frac{\partial}{\partial r}\right)+\frac{L^{2}}{2 m r^{2}} \\
V & =V(r) .
\end{aligned}
$$

The equivalent one-dimensional effective Hamiltonian is

$$
H=-\frac{1}{2 m}\left(\frac{\partial^{2}}{\partial r^{2}}+\frac{2}{r} \frac{\partial}{\partial r}\right)+\left(V(r)+\frac{L^{2}}{2 m r^{2}}\right)
$$

The solutions for unbounded motion belong to the continuous spectrum and have the form

$$
u_{k}(r)=r \psi_{k}(r) \sim e^{-i k r}+\int T\left(k, k^{\prime}\right) \frac{e^{i k^{\prime} r}}{r} d k^{\prime}
$$

with $T\left(k, k^{\prime}\right)$ the offshell scattering amplitude given by he solutions of the integral equation

$$
T\left(k, k^{\prime}\right)=V\left(k, k^{\prime}\right)+G_{0}(k) T\left(k, k^{\prime}\right)
$$


where

$$
V\left(k, k^{\prime}\right)=\int e^{-i k r} V(r) e^{i k^{\prime} r} d r=V\left(k^{\prime}, k\right)
$$

is the potential as a kernel in the momentum variables. The discrete complex values for which there is a solution of the form

$$
u(r)=\int T\left(k, k^{\prime}\right) e^{i k^{\prime} r} d k^{\prime}, \quad u(0)=0
$$

are resonant states and may be included in the complete set of states along with the complex spectrum $\Gamma$. These may be one or more resonant states for the system in addition to any real bound states. Such methods can be used for precision determination of the position and width of resonances.

When the analytic continuation is to the lower half plane the uncovered discrete poles as well as the continuous spectrum have negative imaginary parts so that for positive times $\exp (-i z t)$ has convergence factors $\exp (-|\operatorname{Im} z| t)$. Consequently the survival amplitude decreases in time for positive times and can be computed by integration along the curve in the lower half plane. ${ }^{18}$ For large but not too large positive values of time the contribution of the poles dominate. It therefore appears that the absolute value of $A(t)$ decreases as a function of time. So it appears as if the symmetry with reagard to time has been broken! However we note that for negative times the contribution may not be well calculated this way since the entire spectrum has growing exponentials. Since the spectral measure $d \Pi(\lambda)$ is real along the real axis there is a conjugate analytic continuation to $\mathcal{G}^{*}$ along a complex conjugate contour $\Gamma^{*}$. The spectrum has a positive imaginary part and hence there is exponential decrease with $|t|$ for negative $t$. The resonant states are now at $z_{n}^{*}$. This new representation is appropriate for the amplitude for negative $t$. So for positive times we use one complex spectrum and for negative times we use another. Both senses of time are equivalent but any choice breaks time symmetry.

Instead of the time development being considered for the state vectors we could consider it for the density operators, or more generally thc lincar span of density matrices. The density matrices 
are normalized positive linear sums of projections, or more generally normalized positive linear operators. Since the matrices are to be positive they form a convex set rather than a linear space:

$$
\begin{aligned}
\rho^{+} & =\rho ; \rho \geq 0 ; \operatorname{tr} \rho=1 \\
\cos ^{2} \theta \rho_{1}+\sin ^{2} \theta \rho_{2} & =\rho \geq 0 \text { if } \rho_{1} \geq 0, \rho_{2} \geq 0
\end{aligned}
$$

If $\psi_{n}$ and $\phi_{n}$ are sets of pairs of vectors in the Hilbert space we consider the linear operator $\Sigma a_{n} \psi_{n} \phi_{n}^{+}$which acting on a vector $x$ produces the vector $\Sigma a_{n}\left(\phi_{n}, x\right) \psi_{n}$. These bilinears themselves form a normed vector space with scalar product

$$
\left(\sum_{n} a_{n} \psi_{n} \phi_{n}^{+}, \sum_{m} a_{m}^{\prime} \psi_{m}^{\prime} \phi_{m}^{\prime+}\right)=\sum_{n, m} a_{n}^{*} a_{m}^{\prime}\left(\psi_{n}, \psi_{m}^{\prime}\right)\left(\phi_{m}^{\prime}, \phi_{n}\right)
$$

This Hilbert-Schmidt space is often used as a pre-Hilbert space. If a dense set of vectors $\psi_{n}, \phi_{n}$ are realized as analytic functions of the energy variable the time dependence of

$$
\rho=\sum a_{n} \psi_{n} \phi_{n}^{+}
$$

is given by

$$
\rho(t)=\sum_{n} a_{n} \int_{R^{+}} \psi_{n}(\lambda) \int_{R^{+}} \phi_{n}^{*}\left(\lambda^{\prime}\right) e^{-i \lambda t} d \lambda e^{+i \lambda^{\prime} t} d \lambda^{\prime}
$$

This can be rewritten

$$
\begin{aligned}
\rho(t) & =\sum_{n} a_{n} \int_{R^{+}} d \lambda\left\{\int_{-2 \lambda}^{2 \lambda} \psi_{n}\left(\lambda+\frac{\nu}{2}\right) \phi_{n}^{*}\left(\lambda-\frac{\nu}{2}\right) e^{-i \nu t} d \nu\right\} \\
& =\sum_{n} a_{n} \int_{-\infty}^{\infty} e^{-i \nu t}\left\{\int_{2|\nu|}^{\infty} \psi_{n}\left(\lambda+\frac{\nu}{2}\right) \phi_{n}^{*}\left(\lambda-\frac{\nu}{2}\right) d \lambda\right\} d \nu .
\end{aligned}
$$

This time dependence has an analytic continuation ( when $\psi_{n}, \phi_{n}$ belong to the dense set of analytic vectors) given by

$$
\rho(t)=\sum_{n} a_{n} \int_{R^{+}} d \lambda\left\{\int_{\Gamma_{\lambda}} \psi_{n}\left(\lambda+\frac{z}{2}\right) \phi_{n}^{*}\left(\lambda-\frac{z^{*}}{2}\right) e^{-i z t} d z\right\}
$$

where $\Gamma_{\lambda}$ are open contours from $-2 \lambda$ to $2 \lambda$ lying entirely within the domain of analyticity of the $\left\{\psi_{n}, \phi_{n}\right\}$. In this minimal analytic continuation only $\nu$ is made complex with $\lambda$ remaining real. 
We also note, in passing, that while the spectrum of $\nu$ goes from $-\infty$ to $\infty$, for larger values of $|\nu|$ there is less of the integrand

$$
\int_{2|\nu|}^{\infty} \psi_{n}\left(\lambda+\frac{\nu}{2}\right) \phi_{n}^{*}\left(\lambda-\frac{\nu}{2}\right) d \lambda
$$

We could instead have a non-minimal analytic continuation in which both $\lambda$ and $\nu$ are made complex into $\zeta$ and $z$. Then

$$
\rho(t)=\sum_{n} a_{n} \int_{C} d \zeta\left\{\int_{\Gamma_{\zeta}} \psi_{n}\left(\zeta+\frac{z}{2}\right) \phi_{n}^{*}\left(\zeta^{*}-\frac{z^{*}}{2}\right) e^{-i z t} d z\right\}
$$

where $\Gamma_{\zeta}$ are open contours from $-2 \zeta$ to $+2 \zeta$ with $\zeta$ lying on any complex contour $C$ chosen to go from $-\infty$ to $\infty$. We could write this in the form

$$
\rho(t)=\sum_{n} a_{n} \int_{\Gamma} d z e^{i z t}\left\{\int_{C_{z}} \psi_{n}\left(\zeta+\frac{z}{2}\right) \phi_{n}^{*}\left(\zeta^{*}-\frac{z^{*}}{2}\right) d \zeta\right\}
$$

where $C_{z}$ are contours for $2 z$ to $\infty$ (with $z$ lying on the contour $\Gamma$ from $-\infty$ to $\infty$ ).

The equation of motion of the linear span of the density matrix given by the Liouvillian

$$
i \frac{d \rho}{d t}=\mathcal{L} \rho \equiv[H, \rho]
$$

acts by

$$
\begin{aligned}
i \frac{d \rho}{d t} & =\sum_{n} a_{n} \int_{C} d \zeta\left\{\int_{\Gamma_{\zeta}} z \cdot \psi_{n}\left(\zeta+\frac{z}{2}\right) \phi_{n}^{*}\left(\zeta^{*}-\frac{z^{*}}{2}\right) \cdot e^{-i z t} d z\right\} \\
& =\sum_{n} a_{n} \int_{\Gamma} d z \cdot z \cdot e^{-i z t}\left\{\int \psi_{n}\left(\zeta+\frac{z}{2}\right) \phi_{n}^{*}\left(\zeta^{*}-\frac{z^{*}}{2}\right) d \zeta\right\}
\end{aligned}
$$

If $\left\{\psi_{n}(\lambda)\right\}$ give an analytic basis in $\mathcal{H}$ so that the resolution of the identity

$$
\sum_{n} \psi_{n}(\lambda) \psi_{n}\left(\lambda^{\prime}\right)=\delta\left(\lambda-\lambda^{\prime}\right)
$$

obtains, then an analytic basis for the Hilbert-Schmidt space $\mathcal{K}$ of the bilinears is furnished by $\left\{\psi_{n}(\lambda) \psi_{m}^{*}\left(\lambda^{\prime}\right)\right\}$. The analytic continuations are given by the set $\left\{\psi_{n}\left(\zeta+\frac{z}{2}\right) \psi_{m}^{*}\left(\zeta^{*}-\frac{z^{*}}{2}\right)\right\}$ furnishing 
a basis for the space $\mathcal{J}$ of the bilinears. For any of these basis elements the time dependence and equation of motion are

$$
\begin{array}{r}
\psi_{n}\left(\zeta+\frac{z}{2}\right) \psi_{m}^{*}\left(\zeta^{*}-\frac{z^{*}}{2}\right) \rightarrow \psi_{n}\left(\zeta+\frac{z}{2}\right) \psi_{m}^{*}\left(\zeta^{*}-\frac{z^{*}}{2}\right) e^{-i z t} \\
i \frac{d}{d t}\left\{\psi_{n}\left(\zeta+\frac{z}{2} t\right) \psi_{n}^{*}\left(\zeta^{*}-\frac{z^{*}}{2}, t\right)\right\}=z\left\{\psi_{n}\left(\zeta+\frac{z}{2}, t\right) \psi_{m}^{*}\left(\zeta^{*}-\frac{z^{*}}{2} t\right)\right\} .
\end{array}
$$

When the spectrum of energy is continous these vectors in $\mathcal{J}$, like their primitive counterparts in $\mathcal{G}$ are ideal vectors; and physical states would involve suitable integrals over them.

\section{Scattering}

Let us now consider a quantum system defined in a Hilbert space $\mathcal{H}$ with a Hamiltonian

$$
H=H_{0}+V
$$

where $H_{0}$ is a free Hamiltonian for which we know explicitly the ideal states in the continuum (and the proper eigenvectors for any discrete states). The ideal states are not normalized and are therefore not in $\mathcal{H}$; to get proper states in $\mathcal{H}$ we should take square integrable linear combinations of these ideal states. If $\psi$ is a vector, possibly an ideal vector which is an eigenstate of $H$ and $\psi_{0}$ is a corresponding solution of $H_{0}$ so that

$$
\begin{aligned}
H \psi_{\lambda} & =\lambda \psi_{\lambda} \\
H_{0} \psi_{0 \lambda} & =\lambda \psi_{0 \lambda},
\end{aligned}
$$

then

$$
\left(1-G_{0}(\lambda) V\right) \psi_{\lambda}=\psi_{0 \lambda} ; G_{0}(\lambda)\left(\lambda-H_{0}\right)=1
$$

Hence

$$
\psi_{\lambda}=\left(1-G_{0}(\lambda) V\right)^{-1} \psi_{0 \lambda}=\Omega^{\prime} \psi_{0 \lambda}
$$


which maybe formally written as the power series

$$
\psi=\sum_{n=0}^{\infty}\left(G_{0}(\lambda) V\right)^{n} \psi_{0}
$$

The operator $\Omega^{\prime}$ is a candidate for the Möller ${ }^{19}$ operator $\Omega$ in the sense that

$$
H \Omega^{\prime}=\Omega^{\prime} H_{0}
$$

But $\Omega^{\prime}$ is not necesarily unitary (or even isometric). By a suitable renormalization we can obtain a unitary Möller operator $\Omega$. There are still some questions regarding the spectra of $H$ and $H_{0}$.

The full Green's function $\mathcal{G}(\lambda)$ is given by

$$
\mathcal{G}(\lambda)=\frac{1}{\lambda+i \epsilon-H}=\left(1-G_{0}(\lambda) V\right)^{-1} G_{0}(\lambda) .
$$

By inspection it appears as if the singularities of $\mathcal{G}(\lambda)$ and $G_{0}(\lambda)$ are the same and therefore $H$ and $H_{0}$ are isospectral. This is not necessarily true since $\mathcal{G}(\lambda)$ can have additional singularities from the first factor $\left(1-G_{0}(\lambda) V\right)^{-1}$. This corresponds to not only bound states produced by the interaction but also continuum states in which one of the constituent particles is composite (so that its mass gets shifted). In addition some of the singularities of $G_{0}$ maybe cancelled by the vanishing of $\left(1-G_{0}(\lambda) V\right)^{-1}$. Thus $\mathcal{G}(\lambda)$ and $G_{0}(\lambda)$ may not be, and generally are not, isospectral. The notion of "perturbations vanishing at infinity" leading to a naive asymptotic condition for scattering systems is not valid generally. The new spectra introduced by $\left(1-G_{0} V\right)^{-1}$ do not appear with a canonical weight; this is the cause for the lack of normalization for the Möller operators $\left(1-G_{0} V\right)^{-1}$. This problem is easily corrected by defining

$$
\Omega=\left(1-G_{0} V\right)^{-1} \cdot D^{-1}
$$

where

$$
D^{2}=\left(1-V G_{0}^{+}\right)^{-1} \cdot\left(1-G_{0} V\right)^{-1} .
$$


For nondegenerate states this is a numerical scaling but for degenerate states $\mathcal{D}$ maybe nondiagonal in the degenerate channels.

An important quantity in scattering systems is the off-the-energy shell scattering amplitude defined by

$$
\begin{aligned}
T(\lambda) & =V\left(1-G_{o}(\lambda) V\right)^{-1} \\
& =\sum_{n=0}^{\infty} V\left(G_{o}(\lambda) V\right)^{n}
\end{aligned}
$$

This operator satisfies the integral equation

$$
T(\lambda)=V+G_{o}(\lambda) V T(\lambda)
$$

In terms of $T(\lambda)$ the exact scattering state vector $\Psi_{\lambda}$ may be defined by

$$
\Psi_{\lambda}=\Psi_{o}+\int\left(\Psi_{o \omega}^{+} T(\lambda) \Psi_{o \lambda}\right) G_{o}(\lambda) V \Psi_{o \omega} d \omega
$$

At this point it is convenient to introduce a modified Dirac bra and ket notation for the ideal eigenstates. We denote them by $\mid \lambda \gg$ and $|\lambda\rangle$ so that

$$
H|\lambda \gg=\lambda| \lambda \gg ; H_{0}|\lambda\rangle=\lambda|\lambda\rangle \text {. }
$$

Then $\mid \lambda \gg$ satisfies the integral equation

$$
\begin{aligned}
\mid \lambda \gg & =|\lambda\rangle+\int\left\langle\nu|V| \lambda \gg(\lambda-\nu+i \epsilon)^{-1} \mid \nu\right\rangle d \nu \\
& =|\lambda\rangle+\int\langle\nu|T| \lambda\rangle(\lambda-\nu+i \epsilon)^{-1}|\nu\rangle d \nu .
\end{aligned}
$$

The Möller operator obeys the following relations:

$$
\begin{aligned}
\Omega & =\int d \lambda|\lambda\rangle \ll \lambda\left|, \Omega^{+}=\int d \lambda\right| \lambda \gg\langle\lambda| \\
H_{0} \Omega & =\int d \lambda \lambda|\lambda\rangle \ll \lambda \mid=\Omega H .
\end{aligned}
$$

These considerations can be carried out for analytic Hamiltonians for the generalized dual spaces $\mathcal{G}$ and $\tilde{\mathcal{G}}$. The spectral variable $\lambda$ is now replaced by a complex variable $z$ with possibly 
new thresholdis and/or discrete complex poles appearing. The space $\mathcal{G}$ now has vectors $|z\rangle, \mid z \gg$ with the dual space $\mathcal{G}^{*}$ having $\langle z|, \ll z|$. The equations are just the same as above with $\lambda$ replaced by $z$ with the understanding that it runs over all the spectrum in $\mathcal{G}$. The $i \epsilon$ in the definition of the Green's function is to be understood as the replacement $z \rightarrow z+i \eta$ where $\eta$ is a complex infinitesimal vector in the direction normal to the contours $\Gamma$ in the positive sense. The discrete complex poles $z_{1}$ in $\left(1-G_{0}(z) V\right)^{-1} G_{0}(z)$ now have the time evolution factor $\exp \left(-i z_{1} t\right)$. The dual vector will have the dependence $\left(\exp \left(-i z_{1}^{*} t\right)\right)^{*}=\exp \left(i z_{1} t\right)$ and therefore their product remains unity independent of time.

There is another quantity of interest which is the survival amplitude:

$$
A(t)=\left(\psi, e^{-i H t} \psi\right)=(\psi(0), \psi(t))
$$

For a discrete complex pole

$$
A(t)=\ll z\left|e^{-i z_{1} t}\right| z \gg=\exp \left(-i z_{1} t\right)
$$

and then the survival probability is

$$
P(t)=|A(t)|^{2}=\exp \left(2 \operatorname{Im} z_{1} t\right)
$$

With the poles in the lower half plane

$$
\begin{aligned}
\operatorname{Im} z_{1} & =-\frac{1}{2} \gamma<0 \text { so that } \\
P(t) & =\exp (-\gamma t) .
\end{aligned}
$$

Therefore if we could realize the state corresponding to the complex pole by itself it would die down for positive times. So time symmetry is broken: positive times coresponding to decay.

For negative times the survival probability exceeds unity since it becomes

$$
\exp (-\gamma t)=\exp (\gamma|t|)\rangle 1
$$


This is unsatisfactory and unacceptable. But if we took an analytic continuation into the upper half plane to the space $\mathcal{G}^{*}$ the real analytic nature of the Hamiltonians $H, H_{0}$, and the interaction $V$ there is a complex pole at $z_{1}^{*}$ and for $t<0$ the "survival probability" is less than unity for $t<0$. Though we have talked about single poles, this applies to any complex singularity, isolated or continuous including complex branch points. Thus by taking one or other analytic continutation to $\mathcal{G}$ or $\mathcal{G}^{*}$ we get generalized states with complex energies appropriate for "decaying" states for positive or negative times. Thus both time senses are equivalent but the choice of the appropriate analytic continuation breaks the time symmetry.

The unphysical choice of the discrete poles by themselves in $\mathcal{G}$ and $\mathcal{G}^{*}$ corresponds to the approximation of choosing a real physical state by a state in an "extended energy" space where the energies go from $-\infty$ to $+\infty$. Then the Breit-Wigner energy distribution maybe chosen as the unstable particle "state" for positive times since they give the characteristic decay for the survival probability [3], [4]. For negative times the complex conjugate function maybe chosen. We wil return to this topic in the next section.

\section{Breaking of Time Symmetry}

Given a physical state vector which is a suitable superposition of energy ideal eigenstates of the total Hamiltonian and of the discrete bound states, the state and its survival amplitude maybe written

$$
\begin{aligned}
& |\psi\rangle=\sum a_{n}\left|\lambda_{n} \gg+\int_{0}^{\infty} a(\lambda)\right| \lambda \gg d \lambda ; \lambda_{n}<0 \\
& A(t)=\left\langle\psi\left|e^{-i H t}\right| \psi\right\rangle=\sum\left|a_{n}\right|^{2} e^{-i \lambda_{n} t}+\int_{0}^{\infty}|a(\lambda)|^{2} e^{-i \lambda t} d \lambda
\end{aligned}
$$

In case we choose $a_{n}=0$, the survival amplitude is an analytic function of $t$ analytic in the lower half plane. Then by virture of the Paley-Wiener theorem it has been shown by Khalfin that $A(t)$ cannot be strictly exponential for all values of $t$; for sufficiently large $t$ the exponential behaviour must not obtain. On general grounds of the requirements of threshold behaviour on $a(\lambda)$ we can 
conclude that $A(t)$ should fall off only as an inverse power of $t$.

Can one consider generalized systems in which we have a strictly exponential decay? This would obtain if $a(\lambda)$ is the boundary value of an analytic function $a(z)$ analytic in the upper half plane. Such an $a(\lambda)$ must necessarily be nonzero for $\lambda<0$. We seek a generalized state satifsying

$$
\begin{aligned}
|\phi\rangle & =\int_{-\infty}^{\infty} a(\lambda) \mid \lambda \gg d \lambda \\
A(t) & =\int_{-\infty}^{\infty}|a(\lambda)|^{2} e^{-i \lambda t} d \lambda
\end{aligned}
$$

For strict exponential decay this survival amplitude should get contributions from only a single point $z_{1}$ in the complex contour integral

$$
\int_{C} a(z) a^{*}\left(z^{*}\right) e^{-i z t} d z=\text { const } 2 \pi i e^{-i z_{1} t}
$$

More generally we do have the complex contour integral in the upper half plane.

Such analytic quasi-physical states can be obtained from a physical state by a Hilbert transform:

$$
\left|\phi_{+}\right\rangle=\frac{+1}{2 \pi i} \iint_{0}^{\infty} \frac{a\left(\lambda^{\prime}\right)}{\lambda-\lambda^{\prime}+i \epsilon} \mid \lambda \gg d \lambda^{\prime} d \lambda
$$

A similar state $\left|\phi_{-}\right\rangle$is obtained to represent the quasi-physical state for negative times:

$$
\left|\phi_{-}\right\rangle=\frac{-1}{2 \pi i} \iint_{0}^{\infty} \frac{a\left(\lambda^{\prime}\right)}{\lambda-\lambda^{\prime}-i \epsilon} \mid \lambda \gg d \lambda^{\prime} d \lambda
$$

Clearly

$$
\left|\phi_{+}\right\rangle+\left|\phi_{-}\right\rangle=\left|\phi^{\prime}\right\rangle
$$

is a physical state; and for square integrable states $|\phi\rangle$ th is decomposition is always possible. The survival amplitude is the Fourier transform of a function analytic in the lower half plane; hence $A_{+}(t)$ vanishes for $t<0$ but $\left|A_{+}(t)\right| \ll 1$ for $t>0$. Similarly $A_{-}(t)$ vanishes for $t>0$, but $\left|A_{-}(t)\right|<1$ for $t<0$. 
When the analytic continuation from $\mathcal{H}$ to $\mathcal{G}$ is considered we have a generic state

$$
|\psi\rangle=\int_{\Gamma} a(z)|z\rangle d z+\sum a_{n}\left|z_{n}\right\rangle
$$

with the survival amplitude

$$
A(t)=\int_{\Gamma} a^{*}\left(z^{*}\right) a(z) e^{-i z t} d z+\sum a_{n}^{*} a_{n}
$$

This quantity $A(t)$ is the same as the survival amplitude for $|\psi\rangle$ computed earlier, but now computed by analytic continuation. Hence it cannot be strictly exponential. But the decomposition into the two quasi-states with the variable $z$ running from $-\infty$ to $+\infty$ can be made here also. Construct a complex contour $\Gamma^{\prime}$ from $-\infty$ to $+\infty$ by adjoining a contour $\Gamma_{1}$ from $-\infty$ to 0 and joining it with $\Gamma$. Then we may write

$$
\begin{aligned}
|\psi\rangle & =\left|\phi_{+}\right\rangle+\left|\phi_{-}\right\rangle \\
\left|\phi_{ \pm}\right\rangle & =\frac{ \pm 1}{2 \pi i} \int_{\Gamma^{\prime}}\left\{\int_{\Gamma} \frac{a\left(z^{\prime}\right)}{z-z^{\prime} \pm i \eta} d z^{\prime}+\frac{a_{n}}{z-z_{n}} \pm i \eta\right\}|z\rangle d z
\end{aligned}
$$

Then $\left|\phi_{+}\right\rangle$is analytic by construction above $\Gamma^{\prime}$ and $\left|\phi_{-}\right\rangle$below $\Gamma^{\prime}$. The replacement of $|\psi\rangle$ by $\left|\phi_{ \pm}\right\rangle$for $t \gtrless 0$ breaks time symmetry.

If the generalized quasi-state is given by a sum of two discrete complex states

$$
|\psi\rangle=\left(\left|z_{1}\right\rangle+\left|z_{1}^{*}\right\rangle\right)
$$

then the quasistates $\left|\psi_{ \pm}\right\rangle$are given by

$$
\begin{aligned}
\left|\phi_{+}\right\rangle & =\frac{1}{2 \pi i} \int \frac{1}{z-z_{1}}|z\rangle d z \\
\left|\phi_{-}\right\rangle & =\frac{1}{2 \pi i} \int \frac{1}{z-z_{1}^{*}}|z\rangle d z .
\end{aligned}
$$

The quasi-state $\left|\phi_{+}\right\rangle$has the familiar Beit-Wigner [20] form in its energy dependence. The complex energy state in $\mathcal{G}$ with energy $z_{1}$ is sometimes called the Gamow state. Again we see that for $t \gtrless 0$ we choose two distinct Gamow vectors $\left|z_{1}\right\rangle$ and $\left|z_{1}^{*}\right\rangle$ breaking time symmetry. The quasi-state $\left|\phi_{-}\right\rangle$is the state obtained from $\left|\phi_{+}\right\rangle$by time reversal. 
The Gamow vectors [21] have a pure exponential time dependence and hence furnish a realization of the semigroup of positive time translations:

$$
\begin{aligned}
& T(\tau)\left|\phi_{+}\right\rangle=\left|\phi_{+}, \tau\right\rangle=e^{-i H \tau}\left|\phi_{+}\right\rangle, \tau>0 \\
& T(\tau)\left|\phi_{+}\right\rangle=0 ; \tau<0 .
\end{aligned}
$$

The state $\left|\phi_{-}\right\rangle$furnishes a realization of the semigroup of negative time translations

$$
\begin{aligned}
& T(\tau)\left|\phi_{-}\right\rangle=e^{-i H \tau}\left|\phi_{-}\right\rangle, \tau \leq 0 . \\
& T(\tau)\left|\phi_{-}\right\rangle=0, \tau>0 .
\end{aligned}
$$

What is the most general realization of the time translation semi group?

If we define a generalized state $\left|\Phi, \tau_{0}\right\rangle$ labelled by a positive parameter $\tau_{0}>0$ and linearly dependent on $|\Phi\rangle$ and with the same norm $\langle\tilde{\Phi} \mid \Phi\rangle=1$ with the restriction that $\left|\Phi, \tau_{0}\right\rangle$ vanishes for negative $\tau_{0}$. Then the operations

$$
\begin{aligned}
\mathcal{J}(\tau)\left|\Phi, \tau_{0}\right\rangle & =\left|\Phi, \tau+\tau_{0}\right\rangle \\
\left\langle\tilde{\Phi}, \tau_{0}\right| J(\tau) & =\left\langle\tilde{\Phi}, \tau+\tau_{0}\right|
\end{aligned}
$$

furnish an isometric realization of the time translation semi-group. What is the structure of this realization?

For vectors in the space $\mathcal{H}^{\prime}$ extending $\mathcal{H}$ where the energies are real but unbounded the states

$$
\left|\Phi, \tau_{0}\right\rangle=\int_{-\infty}^{\infty} d \lambda a(\lambda) e^{-i \lambda \tau_{0}} \mid \lambda \gg
$$

satisfy the requirements provided $a(\lambda)$ is analytic in the upper half plane. By a theorem of Titchmarsh, any such function has a Fourier transform which vanishes for negative values of the argument. Then

$$
|a(\lambda)|^{2}=a(\lambda) a^{*}\left(\lambda^{*}\right)
$$


is also the boundary value of an analytic function analytic in the upper half plane. Correspondingly the survival amplitude

$$
\langle\tilde{\phi} \mid \phi\rangle=A(t)=\int_{-\infty}^{\infty} a(\lambda) a^{*}\left(\lambda^{*}\right) e^{-i \lambda t} d \lambda
$$

vanishes for negative values of $t$. For $\left|\phi, \tau_{0}\right\rangle$ the survival amplitude has a larger support

$$
A_{\tau_{0}}(t)=0, t>-\tau_{0} \text {. }
$$

The state $\left|\Psi, \tau_{1}\right\rangle$ may be thought of as having been born at $t=-\tau_{1}$ and developed by the total Hamiltonian from then on. The linear correspondence

$$
J(\tau)\left|\phi, \tau_{1}\right\rangle=\left|\Psi, \tau+\tau_{1}\right\rangle
$$

then furnishes an isometric realization of the semigroup of forward time translations. Any one of these state $\left|\Psi, \tau_{1}\right\rangle$ cai also be time translated backward until $t=-\tau_{1}$. We racognize the compositon law

$$
\begin{gathered}
\mathcal{J}\left(\tau_{2}\right) \mathcal{J}\left(\tau_{1}\right)\left|\Psi, \tau_{0}\right\rangle=\left|\Psi, \tau_{0}+\tau_{1}+\tau_{2}\right\rangle \\
=\mathcal{J}\left(\tau_{2}+\tau_{1}\right)\left|\Psi, \tau_{0}\right\rangle .
\end{gathered}
$$

The operations are realized by only isometries since in general the inverses do not exist.

Given the state $\left|\Psi_{-}\right\rangle$which admit negative time translation semigroup by isometries we have the family

$$
\left|\Psi_{-}, \tau_{0}\right\rangle=\mathcal{J}\left(\tau_{0}\right)\left|\Psi_{-}\right\rangle, \tau_{0}<0
$$

which satisfy

$$
\mathcal{J}\left(\tau_{1}\right)\left|\Psi_{-}, \tau_{0}\right\rangle=\left|\Psi_{-}, \tau_{0}+\tau_{1}\right\rangle, \tau_{1}<0 .
$$

The state $\left|\Psi_{-}, \tau_{0}\right\rangle$ would die at time $t=-\tau_{0}$. This gives us the opportunity to construct the analogue of the classical decaying ensemble. For this purpose construct

$$
|\Psi\rangle=\int_{-\infty}^{0} b(\tau)\left|\Psi_{-}, \tau\right\rangle d z
$$


The time evolution of this state is

$$
\begin{aligned}
T(t)|\Psi\rangle & =\int_{-\infty}^{0} b(\tau)\left|\Psi_{-}, \tau+t\right\rangle d z \\
& =\int_{-\infty}^{0} b(\tau-t)\left|\Psi_{-}, \tau\right\rangle d z
\end{aligned}
$$

So the time evolution on such pseudostates is equivalent to the shift

$$
b(\tau) \rightarrow b(\tau-t)
$$

So a strictly exponential decay could be produced by choosing

$$
b\left(\tau=\exp \left(\frac{\gamma}{2} \tau\right)\right.
$$

so that the amplitude is self similar and the state decreases by $\exp \left(-\frac{\gamma}{2} t\right)$. Any other line shape can also be excited by choosing the amplitude factor $b(\tau)$.

While such a choice may appear artificial this is precisely the skeleton of the Gamow states with strictly exponential decay!

\section{Decaying Pseudo-density Operators}

In the previous sections we studied the time evolution of a quantum system which is described by pure states represented by state vectors. Now we take up the corresponding problems for a mixed state described by density operators. In the Dirac notation if we choose

$$
\rho=\int_{0}^{\infty} \int_{-\frac{\lambda}{2}}^{\frac{\lambda}{2}} \rho(\lambda ; \mu)\left|\lambda+\frac{\mu}{2} \gg \ll \lambda-\frac{\mu}{2}\right| d \lambda d \mu
$$

the time evolution is

$$
\begin{aligned}
\rho(t) & =\int_{0}^{\infty} d \lambda \int_{-\frac{\lambda}{2}}^{\frac{\lambda}{2}} \rho(\lambda ; \mu)\left|\lambda+\frac{\mu}{2} \gg \ll \lambda-\frac{\mu}{2}\right| e^{-i \mu t} d \mu \\
& =\int_{-\infty}^{\infty} e^{-i \mu t}\left\{\int_{2|\nu|}^{\infty} d \lambda \rho(\lambda ; \mu)\left|\lambda+\frac{\mu}{2} \gg \ll \lambda-\frac{\mu}{2}\right|\right\} d \mu .
\end{aligned}
$$


We could rewrite this in the form

$$
\begin{aligned}
\rho(t) & =\rho_{+}(t)+\rho_{-}(t) \\
\rho_{ \pm}(t) & =\int_{0}^{\infty} d \lambda \int_{-\infty}^{\infty}\left|\lambda+\frac{\mu}{2} \gg \ll \lambda-\frac{\mu}{2}\right| e^{-i \mu t}\left\{\int_{-\frac{\lambda}{2}}^{\frac{\lambda}{2}} \frac{ \pm 1}{2 \pi i} \rho\left(\lambda ; \mu^{\prime}\right) \frac{1}{\mu-\mu^{\prime} \pm i \epsilon} d \nu^{\prime}\right\} d \mu .
\end{aligned}
$$

This decomposition has the property

$$
\begin{aligned}
\rho_{ \pm}(t) & =T_{ \pm}(t) \rho_{ \pm}(0), \quad \pm t \geq 0 \\
& =0, \quad \pm t<0
\end{aligned}
$$

with $T_{ \pm}(t)$ furnishing a semigroup realization.

$$
T_{ \pm}\left(t_{1}\right) T_{ \pm}\left(t_{2}\right)=T_{ \pm}\left(t_{1}+t_{2}\right) ; \pm t_{1}, \pm t_{2} \geq 0
$$

Note that while $\rho$ is a physical density matrix $\rho_{+}$and $\rho_{-}$are quasi-physical; they are defined as densities in the space $\mathcal{H}^{\prime}$ extending $\mathcal{H}$ to unphysical negative energies.

With the density operator, the bilinear quantity obtained by taking the trace of the product $\rho(0) \rho(t)$ is the survival probability

$$
P(t)=(\operatorname{tr})(\rho(0) \rho(t))=|A(t)|^{2} .
$$

This is the scalar product in the vector space of linear operators on $\mathcal{H}$. Since

$$
\rho(t)=e^{-i H t} \rho(o) e^{i H t}
$$

and the trace is invariant under cyclic permutations

$$
P(-t)=\operatorname{tr}(\rho(-t) \rho(0))=P(t)
$$

Hence the survival probability is symmetric in time.

Instead of working in the space of linear operators in $\mathcal{H}$ we could analytically continue to the space of linear operators in $\mathcal{G}$ and $\mathcal{G}^{*}$. Then

$$
\begin{aligned}
\rho(t) & =\int_{0}^{\infty}\left\{\int_{-\frac{\lambda}{2}}^{\frac{\lambda}{2}} \rho(\lambda ; z)\left|\lambda+\frac{z}{2} \gg \ll \lambda-\frac{z}{2}\right| e^{-i z t} d z\right\} d \lambda \\
& =\rho_{+}(t)+\rho_{-}(t)
\end{aligned}
$$


with the Hilbert transforms

$$
\rho_{ \pm}(t)=\frac{ \pm 1}{2 \pi i} \int_{0}^{\infty} d \lambda \int_{C^{\prime}}\left\{\int_{-\frac{\lambda}{2}}^{\frac{\lambda}{2}} \rho\left(\lambda, z^{\prime}\right) \frac{1}{z-z^{\prime} \pm i \eta} d z^{\prime}\right\}\left|\lambda+\frac{z}{2} \gg \ll \lambda-\frac{z}{2}\right| e^{-i z t} d z .
$$

The pseudo density matrices $\rho_{ \pm}(t)$ realize the time translation semi groups

$$
\rho_{ \pm}(t)= \begin{cases}T_{ \pm}(t) \rho_{ \pm}(0), & \pm t \geq 0 \\ 0 & , \pm t<0\end{cases}
$$

We now return to scattering systems with the Hamiltonian $H=H_{0}+V$. As before we denote the (possibly ideal) eigenvectors and Green's functions of $H$ and $H_{0}$ in $\mathcal{H}$ by

$$
\begin{aligned}
H \mid \lambda \gg & =\lambda \mid \lambda \gg, G(z)=(z-H)^{-1} \\
H_{0}|\omega\rangle & =\omega|\omega\rangle ; G_{0}(z)=\left(z-H_{0}\right)^{-1} .
\end{aligned}
$$

Then

$$
G(z)=(z-H)^{-1}=\left(1-G_{0}(z) V\right)^{-1} G_{0}(z)
$$

and the (unnormalized) solutions $\mid \lambda \gg$ satisfy

$$
\begin{aligned}
\mid \lambda \gg & \left.=|\lambda\rangle+G_{0}(\lambda) V|\lambda \gg=| \lambda\right\rangle+G_{0}(\lambda) T|\lambda\rangle \\
& =\left\{1+G_{0}(\lambda+i \epsilon) T\right\}|\lambda\rangle
\end{aligned}
$$

with

$$
\begin{aligned}
\langle\omega|T| \lambda\rangle & =\langle\omega|V| \lambda \gg \\
& =\langle\omega|V| \lambda\rangle+\left\langle\omega\left|V G_{0}(\lambda+i \epsilon) T\right| \lambda\right\rangle .
\end{aligned}
$$

The Möller matrix is

$$
\Omega=\int d \lambda|\lambda\rangle \ll \lambda \mid
$$


and satisfies

$$
\begin{aligned}
H_{0} \Omega & =\Omega H, \\
\Omega^{+} \Omega & =\int d \lambda|\lambda \gg \ll \lambda| \\
\Omega^{+} & =\int d \lambda|\lambda\rangle\langle\lambda| .
\end{aligned}
$$

So to the extent that $\{\mid \lambda \gg\}$ and $\{|\lambda\rangle\}$ are complete sets $\Omega^{+} \Omega$ and $\Omega \Omega^{+}$furnish resolutions of the identity and therefore $\Omega$ is not only isometric but unitary.

When we consider analytic Hamiltonians $H, H_{0}$ we can continue the space $\mathcal{H}$ to the dual pair $\mathcal{G}, \tilde{\mathcal{G}}$ and obtain

$$
\begin{aligned}
G(z+i \eta) & =(z-H+i \eta)^{-1}=\left(1-G_{0}(z+i \eta) V\right)^{-1} G_{0}(z+i \eta) \\
\mid z \gg & \left.=|z\rangle+G_{0}(z+i \eta) V\left|z \gg=\left(1+G_{0}(z+i \eta) T\right)\right| z\right\rangle
\end{aligned}
$$

and

$$
\Omega=\int d z|z\rangle \ll z \mid
$$

The possible discrete states satisfy

$$
\left\{1-G_{0}\left(z_{1}\right) V\right\} \mid z_{1} \gg=0
$$

and may therefore be included by writing the terms $\left\{\mid z_{1} \gg \ll z_{1}\right\}$ in the completeness sum. When composite particle continua are included ve may have to renormalize the states by writing

$$
\left.\left|z^{\prime} \gg=N\left(z^{\prime}\right)\left(1+V G_{0}\left(z^{\prime}+i \eta\right) T\right)\right| z^{\prime}\right\rangle
$$

with $N\left(z^{\prime}\right)$ being suitable normalization factors.

When we wish to study mixed states it is convenient and necessary to extend the study to all linear operators in $\mathcal{H}$ or the dual pairs $\mathcal{G}, \tilde{\mathcal{G}}$ rather than just the convex set of Hermitian nonnegative density operators. Given the set of ideal eigenvectors $\{\mid \lambda \gg\}$ and $\{|\lambda\rangle\}$ complete 
sets in the linear space $\mathcal{K}$ of linear operators on $\mathcal{H}$ are given by $\{|\lambda \gg \ll \mu|\}$ and $\{|\lambda\rangle\langle\mu|\}$. The nonnegative hermitian scalar product in $\mathcal{K}$ is given by

$$
\begin{gathered}
\left(|\lambda \gg \ll \mu|, \mid \lambda^{\prime} \gg \ll \mu^{\prime}\right)=\ll \mu\left|\lambda^{\prime} \gg \ll \mu^{\prime}\right| \lambda \gg \\
\left(|\lambda\rangle\left\langle\mu|,| \lambda^{\prime}\right\rangle\left\langle\mu^{\prime}\right|\right)=\left\langle\mu \mid \lambda^{\prime}\right\rangle\left\langle\mu^{\prime} \mid \lambda\right\rangle .
\end{gathered}
$$

The Liouville equations of motion

$$
i \dot{\rho}=[H, \rho]=\mathcal{L} \rho
$$

generalize to these operators by linearity:

$$
i \frac{d}{d t}(|\lambda \gg \ll \mu|)=\mathcal{L}(|\lambda \gg \ll \mu|), .
$$

We write

$$
\begin{aligned}
\mathcal{L} & =\mathcal{L}_{0}+\mathcal{L}_{v} \\
\mathcal{L} & =[H, \cdot], \mathcal{L}_{0}=\left[H_{0}, \cdot\right], \mathcal{L}_{V}=[V, \cdot]
\end{aligned}
$$

Then

$$
\begin{aligned}
\mathcal{L}_{0}|\omega\rangle\left\langle\omega^{\prime}\right| & =\left(\omega-\omega^{\prime}\right)|\omega\rangle\left\langle\omega^{\prime}\right| \\
\mathcal{L}\left|\lambda \gg \ll \lambda^{\prime}\right| & =\left(\lambda-\lambda^{\prime}\right)\left|\lambda \gg \ll \lambda^{\prime}\right| .
\end{aligned}
$$

Then

$$
\begin{aligned}
& \left(|\omega\rangle\left\langle\omega^{\prime}\left|, \mathcal{L}_{0}\right| \lambda \gg \ll \lambda^{\prime}\right|\right) \\
= & \left.\operatorname{tr}\left(\mathcal{L}_{0}\left|\lambda \gg \ll \lambda^{\prime}\right| \omega\right\rangle\left\langle\omega^{\prime}\right|\right)=\left(\omega^{\prime}-\omega\right)\left\langle\omega^{\prime}\left|\lambda \gg \ll \lambda^{\prime}\right| \omega\right\rangle ; \\
& \left(|\omega\rangle\left\langle\omega^{\prime}\left|, \mathcal{L}_{V}\right| \lambda \gg \ll \lambda^{\prime}\right|\right)=-\left\langle\omega^{\prime}\left|\lambda \gg \ll \lambda^{\prime}\right| V \mid \omega\right\rangle\left\langle\omega^{\prime}|V| \lambda \gg \ll \lambda^{\prime} \mid \omega\right\rangle \\
= & T\left(\omega^{\prime}, \lambda\right) \ll \lambda^{\prime}|\omega\rangle-T^{*}\left(\omega, \lambda^{\prime}\right)\left\langle\omega^{\prime}\right| \lambda \gg .
\end{aligned}
$$

But

$$
\left\langle\omega^{\prime}\left|\lambda \gg=\left\langle\omega^{\prime} \mid \lambda\right\rangle+\int T(\mu, \lambda) \frac{d \mu}{\lambda-\mu+i \epsilon}<\omega^{\prime}\right| \lambda\right\rangle
$$


so that

$$
\begin{aligned}
& \left(\omega^{\prime}-\lambda\right)<\omega^{\prime} \mid \lambda \gg=-T\left(\omega^{\prime}, \lambda\right) \\
& \left(\lambda^{\prime}-\omega\right) \ll \lambda^{\prime}|\omega\rangle=-T^{*}\left(\omega, \lambda^{\prime}\right) .
\end{aligned}
$$

Hence

$$
\begin{aligned}
& \left.\left.\operatorname{tr}\left(\mathcal{L}_{0}\left|\lambda \gg \ll \lambda^{\prime}\right| \omega\right\rangle\left\langle\omega^{\prime}\right|\right)=\left(\omega^{\prime}-\lambda+\lambda^{\prime}-\omega+\lambda-\lambda^{\prime}\right)<\omega^{\prime}\left|\lambda \gg \ll \lambda^{\prime}\right| \omega\right\rangle \\
& \left.=\left(\lambda-\lambda^{\prime}\right)<\omega^{\prime}\left|\lambda \gg \ll \lambda^{\prime}\right| \omega\right\rangle-T\left(\omega^{\prime} \lambda\right) \ll \lambda^{\prime}|\omega\rangle-T^{*}\left(\omega \lambda^{\prime}\right)<\omega^{\prime} \mid \lambda \gg .
\end{aligned}
$$

Thus we recover the result

$$
\begin{aligned}
& \left.\left.\operatorname{tr}\left(\left\{\mathcal{L}_{0}+\mathcal{L}_{V}\right\}\left|\lambda \gg \ll \lambda^{\prime}\right| \omega\right\rangle\left\langle\omega^{\prime}\right|\right)=\left(\lambda-\lambda^{\prime}\right)<\omega^{\prime}\left|\lambda \gg \ll \lambda^{\prime}\right| \omega\right\rangle \\
& \left.=\operatorname{tr}\left(\mathcal{L}\left|\lambda \gg \ll \lambda^{\prime}\right| \omega\right\rangle\left\langle\omega^{\prime}\right|\right)
\end{aligned}
$$

as it should be. In particular if we put $\lambda^{\prime}=\lambda$ it follows that

$$
\left(\mathcal{L}_{0}|\lambda \gg \ll \lambda|,|\omega\rangle\left\langle\omega^{\prime}\right|\right)=-\left(\mathcal{L}_{V}|\lambda \gg \ll \lambda|,|\omega\rangle\left\langle\omega^{\prime}\right|\right)
$$

for all values of $\omega, \omega^{\prime}$ and therefore in the limit $\omega^{\prime} \rightarrow \omega$. However if we simply computed $\mathcal{L}_{0}|\omega\rangle\left\langle\omega^{\prime}\right|$ in the limit $\omega^{\prime} \rightarrow \omega$ we get zero. So it appears that

$$
\begin{aligned}
& \left.\operatorname{tr}\left(\left\{\mathcal{L}_{0}+\mathcal{L}_{V}\right\}\left|\lambda \gg \ll \lambda^{\prime}\right| \omega\right\rangle\langle\omega|\right) \\
& \left.=\operatorname{tr}\left(\mathcal{L}_{V}\left|\lambda \gg \ll \lambda^{\prime}\right| \omega\right\rangle\langle\omega|\right)
\end{aligned}
$$

So in the limit $\lambda^{\prime} \rightarrow \lambda$ we get this quantity to be [16]

$$
\begin{gathered}
\left.\operatorname{tr}\left(\mathcal{L}_{V}|\lambda \gg \ll \lambda| \omega\right\rangle\langle\omega|\right) \\
=\langle\omega|V| \lambda \gg \ll \lambda \mid \omega\rangle-\langle\omega|\lambda \gg \ll \lambda| V \mid \omega\rangle
\end{gathered}
$$

which is the matrix element of the "collision operator":

$$
\left(|\lambda \gg \ll \lambda|, \mathcal{L}_{V}|\omega\rangle\langle\omega|\right)=\Theta(\lambda, \omega)
$$


This inconsistency comes in because of the singular nature of the matrix elements of $\mathcal{L}, \mathcal{L}_{0}, \mathcal{L}_{V}$ between the projections $|\lambda \gg \ll \lambda|$ and $|\omega\rangle\langle\omega|$.

The computations go almost verbatim when one goes from the space $\mathcal{K} \sim \mathcal{H} \otimes \mathcal{H}$ to the space $\mathcal{J} \sim \mathcal{G} \otimes \tilde{\mathcal{G}}$

\section{The Invariants of Motion}

The scattering system with Hamiltonian $H=H_{0}+V$ has one constant of motion in $H$ itself (assumed time independent). We saw that

$$
H=\Omega^{+} H_{0} \Omega
$$

By construction $H$ and $H_{0}$ are isospectral. So $H_{0}$ is not necessarily the free Hamiltonian but a suitable companion Hamiltonian with the same spectrum as $H$. The discrete states $\mid \lambda_{1} \gg$ or $\mid z_{1} \gg$ are to be included in the spectrum of $H_{0}$. We shall assume that this has already been done. If $\left\{H_{n}\right\}$ are a complete set of constants of motion of the Hamiltonian $H_{0}$ then

$$
\left\{\Omega^{+} H_{n} \Omega\right\}
$$

are a complete set of constants of motion of the Hamiltonian $H$. If $H_{0}$ describes a system of $N$ free particles with canonical variables $\left\{q_{j}, p_{j} 1 \leq j \leq N\right\}$ there are $2 N-1$ constants of motion which can be expressed in terms of the overcomplete set $\left\{p_{j}, q_{j} p_{k}-q_{k} p_{j}\right\}$. The corresponding overcomplete set of constants of motion for $H$ are

$$
\left\{\Omega^{+} p_{j} \Omega, \Omega^{+}\left(q_{j} p_{k}-q_{k} p_{j}\right) \Omega\right\}
$$

In particular

$$
H=\Omega\left(\frac{\sum p_{j}^{2}}{2 m}\right) \Omega^{+}
$$

While the matrix elements of $\Omega$ are singular this in no way makes the operators singular. (After all the unit operator has the singular matrix elements $\delta\left(\omega-\omega^{\prime}\right)$.) Out of these $2 N-1$ constants 
of motion $N$ are mutually commuting. When the spectrum of $H$ is degenerate the degeneracy index labelling the specific ideal eigenstates is a function of these $N$ commuting constants.

For the analytically continued case in $\mathcal{G}$ we define analogously a comparison Hamiltonian $H_{0}$ isospectral with $H$ by adjoining all the discrete state (and discarding any states of the free Hamiltonian which disappear in the presence of the interaction $V$ ). Then the Möller matrix $\Omega$ and its adjoint $\tilde{\Omega}$ satisfy

$$
\tilde{\Omega} \Omega=1 ; H=\tilde{\Omega} H_{0} \Omega ;
$$

and the constants of motion are given by [22]

$$
\left\{\tilde{\Omega} H_{n} \Omega\right\}
$$

More explicitly

$$
\begin{aligned}
& \ll \tilde{z}^{\prime}\left|\tilde{2} H_{n} \Omega\right| z \gg \\
= & \ll \tilde{z}^{\prime}\left|\left\{1+\int d \mu^{\prime} \frac{\left|\mu^{\prime}\right\rangle\left\langle\mu^{\prime}\right| \tilde{T}}{z^{\prime}-\mu^{\prime}+i \epsilon}\right\} H_{n}\left\{1+\int \frac{d \mu T|\mu\rangle\langle\mu|}{z-\mu-i \epsilon}\right\}\right| z \gg .
\end{aligned}
$$

\section{The Analytic (Continued) Scattering Amplitude}

For a system with a Hamiltonian $H=H_{0}+V$ in a Hilbert space $H$ we constructed a set of ideal eigenstates $\mid \lambda \gg$ of $H$ corresponding to ideal eigenstates $|\lambda\rangle$ of $H_{0}$. In the analytic continutation we have ideal right eigenstates $\mid z \gg$ of $H$ with eigenvalues $z$ and corresponding ideal right eigenstates $|z\rangle$ of $H_{0}$ in $\mathcal{G}$. There are also left ideal eigenvectors $\ll \tilde{z} \mid$ with the same eigenvalues for $H$ and $\langle\tilde{z}|$ for $H_{0}$ in the dual space $\tilde{\mathcal{G}}$. The relationship of $\mid z \gg$ with $|z\rangle$ is given by

$$
\begin{aligned}
\mid z \gg & =\left(1+G_{0}(z) T(z)\right)|z\rangle \\
T(z) & =V+V G_{0}(z) T(z) .
\end{aligned}
$$


The choice of the Green's function $G_{0}(z)$ and hence $\mid z \gg$ is left arbitrary. If we define

$$
G_{0}(z)=\left(z-H_{0}+i \eta\right)^{-1}=G_{0+}(z)
$$

then the corresponding state $\mid z \gg$ is an "in" state appropriate for the scattering with "diverging" scattering waves at $t \rightarrow+\infty$ but none as $t \rightarrow-\infty$. The amplitude of the diverging waves is the scattering amplitude

$$
\left.F(z)=\lim _{z^{\prime} \rightarrow z}\left\langle z^{\prime}\right|\left(z-H_{0}\right)(|z \gg-| z\rangle\right)=\langle z|T(z)| z\rangle
$$

Since $T$ satisfies the integral equation

$$
T(z)=V+V G_{0_{+}}(z) T(z)
$$

we can solve for $T$ in the form

$$
T(z)=\left(1-V G_{0_{+}}(z)\right)^{-1} V
$$

with the formal perturbation series realization

$$
T(z)=V \sum_{0}^{\infty}\left(G_{0_{+}}(z) V\right)^{n}
$$

We immediately get the "unitarity relation on the energy shell":

$$
\begin{aligned}
\lim _{z \rightarrow z^{\prime}}\left\{T^{+}\left(z^{\prime}\right)-T(z)\right\}= & \left(1-V G_{0_{+}}(z)\right)^{-1}\left\{\left(1-V G_{0_{+}}(z)\right) V\right. \\
& \left.-V\left(1-G_{0_{-}}^{+}\left(z^{\prime}\right) V\right)\right\}\left(1-G_{0_{-}}\left(z^{\prime}\right)\right)^{-1} \\
= & \lim _{z \rightarrow z^{\prime}}\left(1-V G_{0_{+}}(z)\right)^{-1}\left\{V G_{0_{-}}^{+}\left(z^{\prime}\right) V-V G_{0_{+}}(z) V\right\}\left(1-G_{0_{+}}\left(z^{\prime} V\right)^{-1}\right. \\
= & \lim _{z \rightarrow z^{\prime}} T(z)\left(G_{0_{-}}^{+}\left(z^{\prime}\right)-G_{0_{+}}(z)\right) T^{+}\left(z^{\prime}\right) \\
= & 2 \pi i \delta\left(z-z^{\prime}\right) T(z) T^{+}\left(z^{\prime}\right) .
\end{aligned}
$$

We could calculate the scattering amplitude $T$ from the $S$-matrix which is the product of the "in" state and the (dual of the) "out" state. We obtain

$$
S\left(z, z^{\prime}\right)=\left\langle z^{\prime}\left|\left(1+G_{0}(z) T(z)\right)\right| z\right\rangle
$$


with

$$
S\left(z, z^{\prime}\right)=\delta\left(z-z^{\prime}\right)\{1+2 \pi i\langle z|T(z)| z\rangle\}
$$

By the unitarity of $T$ on the energy shell we verify immediately that $S$ is unitary:

$$
\begin{aligned}
& \left(1+F^{+}(z) G^{+}(z)\right)\left(1+G_{0}(z) F(z)\right) \\
= & \left.1+F^{+}(z) G_{0}^{+}(z)+G_{0}(z) F(z)+F^{+}(z) G_{0}(z)+G_{0}^{+}(z)\right) F(z)=1 .
\end{aligned}
$$

Considered as an analytic function of $z$, the scattering amplitude $T(z)$ depends not only on the dynamical factor $\left(1-V G_{0+}(z)\right)^{-1}$ but also on $V$. While the poles of $\left(1-V G_{0+}(z)\right)^{-1}$ depends on $V$ it depends also on the "strength" of the potential: when $V$ is replaced by $g V$ where $g$ is a coupling constant, the poles move when $g$ changes. On the other hand the singularities coming from the factor $V$ are "geometrical" in that they do not depend on the magnitude or even the sign of $g$. There are no new states in the spectrum of $H($ in $\mathcal{H}$ or $\mathcal{G}$ ) since that is governed entirely by tile dynamical factor $\left(1-G_{0+}(z) V\right)^{-1}$. Therefore the singularities of the scattering amplitude do not always correspond to the spectrum but maybe "redundant". So care must be taken in identifying the singularities of the scatiering amplitude with the spectrum of physical states though in a field theory the potential has a "geometry" that depends on the spectrum in a crossed channel.

Not only are there redundant singularities but there is also the possibility of the absence of a singularity for certain points ( or sets of points) of the physical spectrum. This occurs when there are discrete states where the (analytically continued) potential has a zero. This gives rise to the possibility of (real or complex energy) states buried in the (real or complex) continuum $[2],[6]$.

Examples of these phenomena are provided by the solvable models described above. For the Lee model

$$
T(z)=\frac{f^{*}\left(z^{*}\right) f(z)}{\alpha(z+i \eta)}
$$


If $f^{*}\left(z^{*}\right) f(z)$ has a pole at $z=z_{2}$ without $\alpha(z)$ vanishing at this point, $z_{2}$ is a redundant pole. If the numerator has a branch point that too would be a redundant singularity. On the other hand if $\alpha(z)$ had a zero at $z=z_{1}$ and $f^{*}\left(z_{1}^{*}\right) f\left(z_{1}\right)=0$ then the scattering amplitude has no pole at $z_{1}$ though there is a discrete state at $z_{1}$ in $\mathcal{G}$.

When $H$ and $H_{0}$ are not isospectral the theory is somewhat more complicated. We see that the set of singularities of the total Green's function consists of all the singularities of $\left(1-G_{0}(z) V\right)^{-1}$ and some of the zeros of $\left(1-G_{0}(z) V\right)^{-1}$ may cancel the singularities of $G_{0}(z)$. For $z$ at one of these values where $1-G_{0}(z) V=0$ we do not have an ideal eigenstate of $H_{0}$, so we have to consider a comparison Hamiltonian $H_{c}$ which has these values of $z$ in its spectrum. We denote the corresponding ideal eigenstates of $H_{c}$ by $\left.\mid z\right)$. Then we may write

$$
\begin{aligned}
\mid z \gg & \left.=\left(1-G_{0}(z) V\right)^{-1} \cdot \mid z\right) \\
& =\mid z)+G_{0}(z) V \mid z \gg
\end{aligned}
$$

There is now no guarantee that this state is normalized. It would definitely not be so if $z$ corresponds to a point eigenvalue (with a normalizable eigenstate) not contained in $H_{0}$ but in $H_{c}$. In addition if such " composite particles" are in any continuum state we may have to renormalize the state: we write

$$
\left.\left|z \gg=Z^{\frac{1}{2}}(z)\left\{|z\rangle+G_{0}(z) V \mid z \gg\right\}=Z^{\frac{1}{2}}(z)\left(1-G_{0}(z) V\right)^{-1}\right| z\right\rangle
$$

where the wavefunction normalization constant $Z(z)$ is to be computed so that $\mid z \gg$ may be normalized. For a nondegenerate discrete state

$$
Z(z)=\left\{\frac{\partial}{\partial z}\left(1-G_{0}(z) V\right)\right\}^{-1}
$$

All the above calculations are carried out for the space $\mathcal{H}$ with $z$ assuming only real values. But all of them can be extended to the generalized dual pairs $\mathcal{G}, \tilde{\mathcal{G}}$ by analytic continuation of the ket and bra vectors in $\mathcal{H}$. The "in" and "out" states correspond to the use of

$$
G_{0 \pm}(z)=\left(z-H_{0} \pm i \eta\right)^{-1}
$$


with $\eta$ as before, being orthogonal to the contour along which the spectrum of $H_{0}$ is defined. Dense sets of kets are continued to provide dense sets in $\mathcal{G}$; dense set of bras are continued to produce dense sets in the dual space $\tilde{\mathcal{G}}$. The action of forming the adjoints of Green's functions is to seek

$$
\tilde{G}(z)=\left(G\left(z^{*}\right)\right)^{*}
$$

The same rule applies to other operators also. Clearly

$$
\tilde{G}_{0+}(z)=G_{0-}(z)
$$

With these basic rules the entire formalism can be applied to the generalized space $\mathcal{G}, \tilde{\mathcal{G}}$ rather than merely to $\mathcal{H}$.

Isospectrality does not necessarily remain valid after analytic continutation. New states maybe adjoined or deleted. Hence the comparison Hamiltonian $H_{c}$ may depend on the particular analytic continuation chosen (and the specific Hamiltonian).

In both $\mathcal{H}$ and in $\mathcal{G}$ when composite particles are formed not only do we have to adjoin point eigenvalues to the spectrum but also involve such composite particles in the many particle scattering sectors also. In any theory which satisfies the cluster decomposition property, that is that when groups of particles are separated from other groups by sufficiently large distances the interactions fall off faster than any inverse power, then the spectrum has "subadditivity". This means that if one group of particles have $z_{1}$ as their total energy and another group has $z_{2}$ as their total energy, then the grand set comprising both the groups of particles have $z=z_{1}+z_{2}$ in their spectrum. (However not all values of $z$ need be of this form: new bound states or new continua may form; hence "subadditivity"). Hence composite particles must occur in scattering states; these states are not in the spectrum of $H_{0}$. We must iherefore have them also added to form $H_{c}$.

In a state involving one composite particle and other noncomposite ("elementary") particles the wave function renormalization is the same as the normalization constant $\left\{\frac{\partial}{\partial z}\left(1-G_{0}(z) V\right)\right\}^{-\frac{1}{2}}$ 
since when the composite particle is "far from the other particles" its own wavefunction renormalization fixes the renormalization for the entire state.

The magnitude of the renormalization constant in more general cases is not known. In particular when we have rearrangement collisions or multiple composite particles the magnitude of $Z$ is unknown.

\section{Continuum Eigenstates, Möller Operators, Renormalization}

Consider the quantum system in $\mathcal{H}$ or in $\mathcal{G}$ with the Hamiltonian

$$
H=H_{0}+V \text {, }
$$

where $H_{0}$ is the "free" Hamiltonian (say, of free particles) for which the states and the spectrum are known. In $\mathcal{H}$ the spectrum of $H_{0}$ is $R^{+}=\{\lambda \mid 0 \leq \lambda<\infty\}$. $H$ may have other eigenvalues, discrete or continuous. Let us formally denote by $|z\rangle$ the ideal eigenvector of $H_{0}$ with the continuum eigenvalue $z$ (real in $\mathcal{H}$, complex in $\mathcal{G}$ ). Let us denote the ideal eigenstate of $H$ by $\mid z \gg$. Then

$$
\begin{aligned}
H_{0}|z\rangle & =z|z\rangle, \\
\left(H_{0}+V\right) \mid z \gg & =H|z \gg=z| z \gg .
\end{aligned}
$$

Choosing $|z\rangle, \mid z \gg$ to have the same continuum normalization, the Möller operator $\Omega$ defined by

$$
\Omega|z\rangle=\mid z \gg
$$

is isometric:

$$
\begin{aligned}
\Omega^{+} \Omega & =1 \text { in } \mathcal{H} \\
\tilde{\Omega} \Omega & =1 \text { in } \mathcal{G} .
\end{aligned}
$$


The state $\mid z \gg$ satisfies the integral equation

$$
\Omega|z\rangle=|z \gg=| z\rangle+G_{0}(z) V \mid z \gg,
$$

where $G_{0}(z)$ is a Green's function for the free Hamiltonian

$$
\left(z-H_{0}\right) G_{0}(z)=1
$$

with singularities at the spectrum of $H_{0}$. Since

$$
\begin{array}{r}
\left.\left(1-G_{0}(z) V\right)|z \gg=| z\right\rangle \\
\left.\left|z \gg=\left(1-G_{0}(z) V\right)^{-1}\right| z\right\rangle .
\end{array}
$$

Define now $\Omega_{1}$ by requiring

$$
\Omega_{1}|z\rangle=\left(1-G_{0}(z) V\right)^{-1}|z\rangle=\mid z \gg .
$$

Note that while $G_{0}(z)$ depends explicitly on $z$, the pre-Möller operator $\Omega_{1}$ does not. The "scattered wave" is

$$
\left.|z \gg-| z\rangle=G_{0}(z) V\left|z \gg=G_{0}(z) V\left(1-G_{0}(z) V\right)^{-1}\right| z\right\rangle,
$$

but to make it physically appropriate we have to use the Sommerfield outgoing wave condition:

$$
G_{0}(z) \rightarrow G_{0+}(z)=\left(z-H_{0}+i \epsilon\right)^{-1}, \epsilon \rightarrow 0^{+} .
$$

This scattered wave corresponds to a source amplitude $A(z)$ given by

$$
\left.A(z)|z\rangle=\left(z-H_{0}\right)(|z \gg-| z\rangle\right)
$$

which is seen to be

$$
A(z)=V\left(1-G_{0}(z) V\right)^{-1}
$$


These results are exact but do not deal with all states since $\left(1-G_{0}(z) V\right)$ can vanish at values not in the spectrum of $H_{0}$. We shall return to this question later in this section.

These results can be obtained from a formal perturbation theory [13]. The solution $\mid z \gg$ can be expressed in the form of a power series:

$$
\begin{aligned}
\mid z \gg_{1} & =\left\{1+G_{0}(z) V+G_{0}(z) V G_{0}(z) V+\cdots\right\}|z\rangle=\left\{\sum_{0}^{\infty}\left(G_{0}(z) V^{n}\right\} \mid z\right. \\
\Omega_{1}|z\rangle & =\left\{\sum_{0}^{\infty}\left(G_{0}(z) V\right)^{n}\right\}|z\rangle \\
T(z) & =V+V G_{0}(z) T(z)=V \cdot\left\{\sum_{0}^{\infty}\left(G_{0}(z) V\right)^{n}\right\} \\
S(z) & =1+2 \pi i T(z) .
\end{aligned}
$$

We observe

$$
\begin{aligned}
H \sum_{0}^{\infty}\left(G_{0}(z) V\right)^{n}|z\rangle & =\left\{z-\left(z-H_{0}\right)+V\right\} \sum_{0}^{\infty}\left(G_{0}(z) V\right)^{n}|z\rangle \\
& =\left\{z+\left(V-G_{0}^{-1}(z)\right)\right\} \sum_{0}^{\infty}\left(G_{0}(z) V\right)^{n}|z\rangle \\
& =z \sum_{0}^{\infty}\left(G_{0}(z) V\right)^{n}|z\rangle .
\end{aligned}
$$

Since $\left.\left|z \gg_{1}=\sum_{0}^{\infty}\left(G_{0}(z) V\right)^{n}\right| z\right\rangle$ is an (ideal) eigenstate of $H$ corresponding to $|z\rangle$ for $H_{0}$ it follows that

$$
\left.\Omega_{1}|z\rangle=\left|z \gg_{1}=\left\{\sum_{0}^{\infty}\left(G_{0}(z) V\right)^{n}\right\}\right| z\right\rangle .
$$

We have not yet guaranteed that $\mid z \gg_{1}$ is normalized nor even if it is non-zero. In fact at precisely the zeros of

$$
\left(1-G_{0}(z) V\right)^{-1}=\sum_{0}^{\infty}\left(G_{0}(z) V\right)^{n}
$$

the state $\mid z \gg_{1}$ vanishes! In the generic case we must normalize $\mid z \gg_{1}$ to obtain the proper state $\mid z \gg$. For this purpose we compute the non-negative matrix $Z=\Omega_{1} \Omega_{1}^{+}$. Then

$$
\Omega=Z^{-\frac{1}{2}} \Omega_{1}
$$


satisfies

$$
\Omega \Omega^{+}=Z^{-1} \Omega_{1} \Omega_{1}^{+}=1
$$

This construction is possible only when $Z$ is non-singular. When $Z$ is singular we define $Z^{-\frac{1}{2}}$ as the generalized inverse computed in the non-null subspace of $Z$; if the projection to the null space is $\mathcal{P}_{0}$, then

$$
Z^{-1}=\left\{\left(1-\mathcal{P}_{0}\right) Z\left(1-\mathcal{P}_{0}\right)\right\}^{-1}
$$

The Möller matrix so defined is isometric but not unitary:

$$
\begin{array}{r}
\Omega^{+} \Omega=1, \quad \Omega \Omega^{+}=1-\mathcal{P}_{0} \\
\Omega_{1} \Omega_{1}^{+}|z\rangle=Z|z\rangle=\sum_{m, n=0}^{\infty}\left(V G^{+}(z)\right)^{m} \cdot(G(z) V)^{n}
\end{array}
$$

which vanishes only where

$$
\sum_{0}^{\infty}\left(G_{0}\left(z_{1}\right) V\right)^{n}=\left(1-G_{0}\left(z_{1}\right) V\right)^{-1}
$$

has a zero. When such zeros obtain there is a change in the spectrum of $H$; these values of $z_{1}$ are no longer in the spectrum of $H$. In addition wherever $\left(1-G_{0}(z) V\right)^{-1}$ diverges, that is wherever

$$
G_{0}(\zeta) V=1
$$

for some value $z=\zeta$ there are new states in $H$ which were not in $H_{0}$.

The new states $\mid \zeta \gg$ should be adjoined to the states $\mid z \gg$ to form a complete set of states and there are no states $\mid z_{1} \gg$. This spectrum of $H$ is to be associated with the comparison Hamiltonian $H_{c}$. We can define a unitary Möller matrix on these states:

$$
\Omega^{+} \Omega=\Omega \Omega^{+}=1
$$

As stated in the previous section these considerations apply equally to the perturbation theory in $\mathcal{G}$. We need to define

$$
G_{0}(z)=\left(z-H_{c}+i \eta\right)^{-1}
$$


and replace adjoints by the duals and obtain:

$$
\begin{aligned}
\Omega_{1}|z\rangle & =\sum_{n=0}^{\infty}\left(G_{0}(z) V\right)^{n}|z\rangle \\
Z & =\Omega_{1} \tilde{\Omega}_{1} \\
\tilde{\Omega}_{1} & =\sum_{m=0}^{\infty}\left(V \tilde{G}_{0}(z)\right)^{m}
\end{aligned}
$$

and

$$
\Omega=Z^{-\frac{1}{2}} \Omega_{1} .
$$

If we start with the free Hamiltonian the spectrum of $H$ maybe different. The places where $G_{0}\left(z_{1}\right)$ takes the value unity are to be deleted from the spectrum of $H_{0}$ and the places where

$$
\sum_{0}^{\infty}\left(G_{0}(\zeta) V\right)^{n}
$$

diverges are to be added to the spectrum. So we get a new comparison Hamiltonian $H_{c}$ which is "free" and isospectral with $H$.

$$
\begin{array}{r}
H_{c}|z\rangle=z|z\rangle ;\{z\} \neq\left\{z_{1}\right\} . \\
H_{c}|\zeta\rangle=\zeta|\zeta\rangle
\end{array}
$$

The "augmented Möller operator" intertwines $H$ and $H_{c}$ and is unitary:

$$
\Omega H_{c}=H \Omega ; \Omega \Omega^{+}=\Omega^{+} \Omega=1 .
$$

\section{Perturbation Theory in Generalized Spaces: Solvable Examples}

Let us now apply this formalism to certain concrete examples: The one-channel Yamaguchi separable potential model [23] (for S-waves) has a state space of functions $f(z)$ which are $L_{2}(0, \infty)$ with the (norm $)^{2}$ given by

$$
\|f\|^{2}=\int_{0}^{\infty} f^{*}\left(x^{*}\right) f(x) d x .
$$


We have removed inessential kinematic factors to simplify computations and focus on the essential structure; note that the integration variable is the free particle relative energy rather than the relative momentum. An ideal basis is given by the continuum normalized function realization.

$$
|z\rangle=\delta(z-x)
$$

so that

$$
|f\rangle=\int_{0}^{\infty} f(z)|z\rangle d z
$$

is a normalizable state. We choose $z$ or $x$ to be the energy variable so that

$$
H_{0}|z\rangle=z|z\rangle
$$

Take the separable potential interaction to get $\left(H_{0}+V\right)|f\rangle=H|f\rangle=H_{0}|f\rangle: t\langle f \mid g\rangle|f\rangle$. We may represent these Hamiltonians formally as matrices:

$$
H=H_{0}+V=z \delta\left(z-z^{\prime}\right) \pm g(z) \tilde{g}\left(z^{\prime}\right)
$$

Then

$$
\begin{aligned}
G_{0}(\lambda) V & = \pm(\lambda-z+i \eta)^{-1} g(z) \tilde{g}\left(z^{\prime}\right) \\
\left(G_{0}(\lambda) V\right)^{2} & =g(z) \tilde{g}\left(z^{\prime}(\lambda-z+i \eta)^{-1} \frac{\tilde{g}\left(z^{\prime \prime}\right) g\left(z^{\prime \prime}\right)}{\lambda-z^{\prime \prime}+i \eta} d z^{\prime \prime}\right.
\end{aligned}
$$

Then

$$
\begin{aligned}
\sum_{0}^{\infty}\left(G_{0}(\lambda) V\right)^{n} & =\delta\left(z-z^{\prime} 1 \pm\left\{(\lambda-z+\cdot i \eta)^{-1} g(z) g(z) 1+\int \frac{\tilde{g}\left(z^{\prime \prime}\right) g\left(z^{\prime \prime}\right)}{\lambda-z^{\prime \prime}+i \eta} d z^{\prime \prime}\right.\right. \\
& =\delta\left(z-z^{\prime}\right) \pm\left\{\frac{g(z) \tilde{g}\left(z^{\prime}\right)}{(\lambda-z+i \eta) \beta(\lambda+i \eta)}\right\}
\end{aligned}
$$

where

$$
\beta(\lambda+i \eta)=1 \pm \int \frac{\tilde{g}\left(z^{\prime \prime}\right) g\left(z^{\prime \prime}\right) d z^{\prime \prime}}{\lambda-z^{\prime \prime}+i \eta}
$$


is a real analytic function with a cut along the spectrum of $H_{0}$ (the real line for 0 to $\infty$ for $\mathcal{H}$, a coniplex contour from 0 to $\infty$ for $\mathcal{G}$ ). Since $\beta(z)$ is finite for all $z$ it follows that

$$
\Omega_{1}|z\rangle=\sum_{0}^{\infty}\left(G_{0}(z) V\right)^{n}|z\rangle
$$

cannot vanish for any $z$ and hence the entire spectrum of $H_{0}$ is included in the spectrum of $H$. However if $\sum_{0}^{\infty}\left(G_{0}(z) V\right)^{n}$ can become equal to unity so that $\beta(z)$ is zero for any $z$ in the cut plane there will be one or more additional states $|\zeta\rangle$ in $H$ and then $H_{c}$ will be different from $H_{0}$. A more detailed study of $\beta(z)$ shows that it can vanish at most only at one real point for the negative sign in (9.2), that is for an attractive potential.

The ideal eigenstates $\mid z \gg$ are given by

$$
\left\langle z^{\prime}\right| z \gg=\delta\left(z^{\prime}-z\right) \pm \frac{g\left(z^{\prime}\right) \tilde{g}(z)}{\left(z-z^{\prime}+i \eta\right) \beta(z)}
$$

and are continuum normalized:

$$
\ll z^{\prime} \mid z \gg=\delta\left(z^{\prime}-z\right)
$$

However when a bound state $|\zeta\rangle$ arises as the isolated zero of $\beta(z)$ the unnormalized solution is:

$$
\left\langle z^{\prime}\right| \zeta \gg_{1}=\frac{\tilde{g}\left(z^{\prime}\right)}{\left(\zeta-z^{\prime}\right)}
$$

and the state gets normalized by $Z^{-\frac{1}{2}}$ with

$$
Z=\int \frac{\tilde{g}\left(z^{\prime}\right) g\left(z^{\prime}\right) d z^{\prime}}{\left(\zeta-z^{\prime}\right)^{2}}=\beta^{\prime}(\zeta)
$$

Hence

$$
\left\langle z^{\prime}\right| \zeta \gg=\left(\beta^{\prime}(\zeta)\right)^{-\frac{1}{2}} \frac{\tilde{g}\left(z^{\prime}\right)}{\zeta-z^{\prime}} .
$$

The augmented Möller matrix elements are

$$
\begin{aligned}
\left\langle z^{\prime}|\Omega| z\right\rangle & =\delta\left(z^{\prime}-z\right) \pm \frac{g\left(z^{\prime}\right) \tilde{g}(z)}{\left(z-z^{\prime}+i \eta\right) \beta(z)} \\
\langle\zeta|\Omega| z\rangle & =\left(\beta^{\prime}(\zeta)\right)^{-\frac{1}{2}} \frac{\tilde{g}(z)}{\zeta-z}
\end{aligned}
$$


This augmented matrix can be verified to be unitary. One can get modified models by replacing $V$ by

$$
\left\langle z^{\prime}|V| z\right\rangle=\sum_{r=1}^{N} \pm g_{r}\left(z^{\prime}\right) \tilde{g}_{r}(z)
$$

Then there is the possibility of many discrete points in the spectrum of $H$. But all points in the spectrum of $H_{0}$ are still in $H$.

For a simple example of the deletion from the spectrum of $H_{0}$ to obtain the spectrum of $H$ we take the Lee model [9]. In this case the vector space is the space of a pair of complex number and $a$ function in the form

$$
\left(f_{0}, f(z)\right)
$$

with the $(\text { norm })^{2}$ given by

$$
\|f\|^{2}=f_{0}^{*} f_{0}+\int_{0}^{\infty} f^{*}\left(z^{*}\right) f(z) d z
$$

The Hamiltonian $H_{0}$ has a discrete eigenvalue $m_{0}$ and a continuous spectrum from 0 to $\infty$. The total Hamiltonian is defined by

$$
\begin{aligned}
(H f)_{0} & =m_{0} f_{0}+\int g^{*}\left(z^{* \prime}\right) f\left(z^{\prime}\right) d z^{\prime} \\
(H f)(z) & =g(z) f_{0}+z f(z) .
\end{aligned}
$$

The matrix form of the Hamiltonian is

$$
H=\left(\begin{array}{cc}
m_{0} & f^{*}\left(z^{* \prime}\right) \\
f(z) & z \delta\left(z-z^{\prime}\right)
\end{array}\right)
$$

Then

$$
G_{0}(\lambda) V=\left(\begin{array}{cc}
0 & \frac{g^{*}\left(z^{* \prime}\right)}{\lambda-m_{0}} \\
\frac{g(z)}{\lambda-z+i \eta} & 0
\end{array}\right)
$$


and

$$
\left(G_{0}(\lambda) V\right)^{2}=\left(\begin{array}{cc}
\left(\lambda-m_{0}\right)^{-1} \int d z^{\prime \prime} \frac{f^{*}\left(z ^ { * \prime \prime } f \left(z^{\prime \prime}\right.\right.}{\lambda-z^{\prime \prime}+i \eta} & 0 \\
0 & \frac{f(z) f^{*}\left(z^{\prime *}\right)}{(\lambda-z+i \eta)\left(\lambda-m_{0}\right)}
\end{array}\right)
$$

Hence

$$
\begin{gathered}
\sum_{n=0}^{\infty}\left(G_{0}(\lambda) V\right)^{2 n}=\left(\begin{array}{cc}
\frac{\left(\lambda-m_{0}\right)}{\alpha(\lambda+i \eta)} & 0 \\
0 & \delta\left(z^{\prime}-z\right)+\frac{f^{* \prime}\left(z^{* \prime} f(z)\right.}{(\lambda-z+i \eta) \alpha(\lambda+i \eta)}
\end{array}\right) \\
\sum_{n=0}^{\infty}\left(G_{0}(\lambda) V\right)^{2 n+1}=\left(\begin{array}{cc}
0 & \frac{f^{*}\left(z^{\prime *}\right)}{\alpha(\lambda+i \eta)} \\
\frac{f(z)\left(\lambda-m_{0}\right)}{(\lambda-z+i \eta) \alpha(\lambda+i \eta)} & 0
\end{array}\right) .
\end{gathered}
$$

with

$$
\alpha(\zeta)=\zeta-m_{0}-\int \frac{f^{*}\left(z^{* \prime \prime}\right) f\left(z^{\prime \prime}\right)}{\lambda-\zeta} d z^{\prime \prime}
$$

Hence

$$
\sum_{n=0}^{\infty}\left(G_{0}(\lambda) V\right)^{n}=\left(\begin{array}{cc}
\frac{\left(\lambda-m_{0}\right)}{\alpha(\lambda+i \eta)} & \frac{f^{*}\left(z^{\prime *}\right)}{\alpha(\lambda+i \eta)} \\
\frac{f(z)\left(\lambda-m_{0}\right)}{(\lambda-z+i \eta) \alpha(\lambda+i \eta)} & \delta\left(z^{\prime}-z\right)+\frac{f^{*}\left(z^{\prime *}\right) f(z)}{(\lambda-z+i \eta) \alpha(\lambda+i \eta)}
\end{array}\right)
$$

This operator becomes singular for $\lambda=m_{0}$ and hence this point in the spectrum of $H_{0}$ is absent in the spectrum of $H$ and hence of $H_{c}$.

As to augmenting the spectrum we look for poles of $\sum_{0}^{\infty}\left(G_{0}(\lambda) V\right)^{n}$. In this model there is at most one real location for $\alpha(\lambda)$ to vanish in $\mathcal{H}$. If there is a zero

$$
\alpha(M)=0
$$

then this point $\zeta=M$ should be added to the spectrum so that $H_{c}$ has $\zeta=M$ and $0<\zeta<\infty$ for the spectrum in $\mathcal{H}$. In $\mathcal{G}$ there is always a zero for real $M$ or complex $\zeta$ where $\alpha(\zeta)=0$. 
In all these cases the continuum solutions

$$
\left.\left|z \gg=\Omega_{1}\right| z\right\rangle=\sum_{0}^{\infty}\left(G_{0}(z) V\right)^{n}|z\rangle
$$

are normalized. But for the discrete solution we need to renormalize the $\Omega_{1}$ matrix elements by the factor $Z^{-\frac{1}{2}}$ with

$$
Z=\alpha^{\prime}(\zeta)
$$

In both the Yamaguchi and Lee models the continuum remains unchanged. A model in which the continuous spectrum also shifts is provided by the Cascade model [12]. In this the state vectors may be realized by a triplet $(\mathcal{X}, \phi(\omega), \psi(\omega, \nu))$ with $(\text { norm })^{2}$ given by

$$
\chi^{*} \chi+\int \phi^{*}\left(\omega^{*}\right) \phi(\omega) d \omega+\iint \psi^{*}\left(\omega^{*}, \nu^{*}\right) \psi(\omega, \nu) d \omega d \nu
$$

The matrix realization of the Hamiltonian is:

$$
H=\left(\begin{array}{ccc}
m_{0} & f^{* \prime}\left(\omega^{\prime \prime \prime}\right) & 0 \\
f(\omega) & \left(m_{1}+\omega\right) \delta\left(\omega^{\prime}-\omega\right) & g^{*}\left(\nu^{\prime \prime} \delta\left(\omega-\omega^{\prime}\right)\right. \\
0 & g(\nu) \delta\left(\omega-\omega^{\prime}\right) & (\omega+\nu) \delta\left(\omega^{\prime}-\omega\right) \delta\left(\nu^{\prime}-\nu\right)
\end{array}\right)
$$

Then

$$
G_{0}(z) V=\left(\begin{array}{lcc}
0 & \left(z-m_{0}\right)^{-1} f^{*}\left(\omega^{\prime}\right) & 0 \\
\left(z-m_{1}-\omega\right)^{-1} f(\omega) & 0 & \left(z-m_{1}-\omega\right)^{-1} \delta\left(\omega-\omega^{\prime}\right) g^{*}\left(\nu^{* \prime}\right) \\
0 & (z-\omega-\nu)^{-1} g(\nu) \delta\left(\omega-\omega^{\prime}\right) & 0
\end{array}\right)
$$

and hence

$$
\left(\begin{array}{ccc}
\left(z-m_{0}\right)^{-1} \int \frac{f^{*}\left(x^{*}\right) f(x) d x}{z-x+i \eta} & 0 & \frac{f^{*}\left(\omega^{* 1}\right) g^{*}\left(\nu^{* 1}\right)}{\left(z-m_{0}\right)\left(z-m_{1}-\omega^{\prime}\right)} \\
0 & \frac{f(\omega) f^{*}\left(\omega^{*^{\prime}}\right)}{\left(z-m_{0}\right)\left(z-m_{1}-\omega\right)}+ & 0 \\
\frac{f\left(\omega^{\prime}\right) g(\nu)}{\left(z-m_{1}-\omega\right)} \int \frac{g^{*}\left(y^{*}\right) g(y) d y}{(z-\omega-y)} & \frac{\delta\left(\omega-\omega^{\prime}\right) g(\nu) g^{*}\left(\nu^{* \prime}\right)}{(z-\omega-\nu)\left(z-m_{1}-\omega\right)}
\end{array}\right) \text {. }
$$


If the wavefunction has the form

$$
\psi_{z}=\left(\begin{array}{c}
a(z) \\
b(z, \omega) \\
c(z, \omega, \nu)
\end{array}\right)
$$

satisfying

$$
G_{0}(z) V \psi_{z}=\psi_{z}
$$

then $b(z, \omega)$ satisfies

$$
\begin{aligned}
& \left(z-m_{0}\right)^{-1}\left(z-m_{1}-\omega\right)^{-1} f(\omega) \int f^{*}\left(\omega^{*^{\prime}}\right) b\left(z, \omega^{\prime}\right) d \omega^{\prime} \\
& +\left(z-m_{1}-\omega\right)^{-1} b(z, \omega) \int \frac{g^{*}\left(y^{*}\right) g(y) d y}{z-\omega-y}=b(z, \omega)
\end{aligned}
$$

or

$$
\begin{aligned}
& \left\{\left(z-m_{1}-\omega\right)-\int \frac{g^{*}\left(y^{*}\right) g(y) d y}{z-\omega-y}\right\} b(z, \omega)=\frac{f(\omega)}{z-m_{0}} \int f^{*}\left(\omega^{*}\right) b\left(z, \omega^{\prime}\right) d \omega^{\prime} \\
& \equiv \gamma(z-\omega) b(z, \omega) .
\end{aligned}
$$

If $\gamma(z)$ has a zero at $z=\mu$ then the generic solution is

$$
b(z, \omega)=\delta(z-\mu-\omega)+\frac{f(\omega)}{(z-\omega)\left(z-m_{0}\right)} \int f^{*}(x) b(z, x) d x
$$

This integral equation can be solved by standard means:

$$
\begin{aligned}
\int f^{*}\left(x^{*}\right) b(z, x) d x & =f^{*}(z-\mu)+\frac{1}{z-m_{0}} \int \frac{f^{*}\left(y^{*}\right) f(y)}{\gamma(z-y)} d y \cdot \int f^{*}\left(x^{*}\right) b(z, x) d x \\
& =\frac{f^{*}\left(z^{*}-\mu^{*}\right)\left(z-m_{0}\right)}{\left\{z-m_{0}-\int \frac{f^{*}\left(z^{*}\right) f(y) d y}{\gamma(z-y)}\right\}}=\frac{\left(z-m_{0} f^{*}\left(z^{*}-\mu^{*}\right)\right.}{\alpha(z)}
\end{aligned}
$$

and, hence

$$
b(z, \omega)=\delta(z-\mu-\omega)+\frac{f(\omega)}{\gamma(z-\omega)} \frac{f^{*}\left(z^{*}-\mu^{*}\right)}{\alpha(z)} .
$$

The effect of the interaction is to shift the continuum from $m_{1}<\omega<\infty$ to $\mu<\omega<\infty$ and possibly to introduce the zero of $\alpha(z)$ as a discrete state. 
In this case finding the spectrum of $\sum_{0}^{\infty}\left(G_{0}(\lambda) V\right)^{n}$ and the ideal eigenfunctions has been done in an earlier paper [12]. The spectrum consists of an infinitely degenerate continuum $0<\lambda<\infty$, possibly a nondegenerate continuum $m<\lambda<\infty(m<0)$, and possibly a discrete state at $\lambda=M$ if

$$
\begin{aligned}
& \alpha(z)=z-m_{o}-\int \frac{f^{*}\left(\omega^{*}\right) f(\omega) d \omega}{\gamma(z-\omega)} \\
& \gamma(z)=z-m_{1}-\int \frac{g^{*}\left(\nu^{*}\right) g(\nu) d \nu}{z-\nu}
\end{aligned}
$$

have zeros:

$$
\begin{aligned}
& \alpha(M)=0 ; \quad M<0 ; \\
& \gamma(m)=0 ; \quad m<0 .
\end{aligned}
$$

Thus the nondegenerate continuum $m_{1}<\lambda<\infty$ is eliminated; and $\lambda=m_{0}$ is also eliminated from the spectrum of $H_{o}$. If $\alpha(z)$ has a discrete zero there is a discrete eigenvalue $\lambda=M$ augmenting the spectrum. If $f(z)$ has a discrete zero there is a nondegenerate continuum from $m<\lambda<\infty$.

Even more interesting is the wavefunction renormalizations for the states. The infinitely degenerate continuum $0<\lambda<\infty$ needs no renormalization. The discrete state $\lambda=M$ has the wavefunction renormalization $Z_{M}^{-\frac{1}{2}}$ with

$$
Z_{M}=\alpha^{\prime}(M)
$$

The continuum $m<\lambda<\infty$ has the wavefunction renormalization $Z^{-\frac{1}{2}}$ with

$$
Z=\alpha^{\prime}(m)
$$

This need for renormalization of the continuum channel shows the limits of validity of traditional scattering theory where the continuum channels have no renormalization by invoking the adiabatic hypothesis. The "persistent interaction" creating a composite particle of mass $m$ does not obey adiabatic decoupling and hence the failure of the usual asymptotic condition. 
When the "bare mass" parameters $m_{0}, m_{1}$ are suitably increased the eigenvalues $\lambda=m$ and $\lambda=M$ disappear; and in $\mathcal{H}$ there are no discrete state or nondegenerate continuum. But by analytic continuation the bound states are realized at complex values of $m, M$. In this case the nondegenerate continuum from $m$ to $\infty$ is along a complex contour; and has the wave function renormalization already derived. The results can be obtained from the formal perturbation series in the analytically continued theory.

Richer solvable models with more than one shifted continuum and the possibility of rearrangement collisions are currently under study with a view to seeing explicitly the realization of the ideas discussed in this Section.

\section{Evolution of the Density Operators}

The discussion in the previous sections have been in terms of state vectors and linear operators acting on the vectors. This corresponds to the special case of pure states and the expectation value of a dynamical variable is sesquilinear in the state. A framework including mixed states can be obtained by using density operators $\rho$. For a dynamical variable $A$ and a trace class density operator $\rho$ the expectation value is

$$
<A>_{\rho}=\operatorname{tr}(A \rho)
$$

More generally we can consider the algebra of dynamical variables and nonnegative normalized linear functions $\mathcal{L}$ on them:

$$
\begin{aligned}
A & \rightarrow \mathcal{L}(A) \\
1 & \rightarrow \mathcal{L}(1)=1 \\
A^{+} A & \rightarrow \mathcal{L}\left(A^{+} A\right) \geq 0 .
\end{aligned}
$$

Being an algebra $\mathcal{A}$ is a vector space; the linear functionals $\mathcal{L}$ on $\mathcal{A}$ constitute elements of the dual space with the state functionals, by virtue of their being nonnegative and normalized, constitute 
a convex set.

The most general dynamical evolution on $\mathcal{A}$ implemented by linear automorphisms is projectively equivalent to a Hamiltonian evolution; and may be alternately realized by unitary transformations on the density operator.

The action of the Hamiltonian time development on the density operator is according to

$$
i \dot{\rho}=[H, \rho]=\mathcal{L}_{H} \rho
$$

where $\mathcal{L}$ is a superoperator acting on the vector space of density operators and their span. The integrated form of the equation of motion is

$$
\rho(t)=\exp \left(-i t \mathcal{L}_{H}\right) \cdot \rho(0)
$$

If we could find the spectrum of $\mathcal{L}_{H}$ then we could seek a resolution of the identity and an integral representation of the time development. If

$$
\mathcal{L}_{H} R_{\lambda}=\lambda R_{\lambda}
$$

and $A^{\lambda}$ is dual to $R_{\lambda}$, so that

$$
\left(A^{\mu}, R_{\lambda}\right)=\operatorname{tr}\left(A^{\mu} R_{\lambda}\right)=\delta_{\lambda}^{\mu}
$$

and

$$
\int d \lambda R_{\lambda} A^{\lambda}=\mathbf{1}
$$

then

$$
\rho(t)=\int \operatorname{tr}\left(A^{\mu} \rho\right) \cdot R_{\mu} e^{-i \mu t} d \mu
$$

Note that the integrand is a multiple of $R_{\lambda}$ which need not be a density operator and for $\lambda \neq 0$ it will have trace zero. It is therefore important to determine the spectrum of $\mathcal{L}_{H}$. When we 
realize the density operator $\rho$ as being sesquilinear in the state vectors the spectrum of $\mathcal{L}_{H}$ is the difference between points in the spectrum of $H$ :

$$
\mu=\lambda_{1}-\lambda_{2}
$$

By writing

$$
\lambda_{1}=\nu+\frac{\mu}{2}, \lambda_{2}=\nu-\frac{\mu}{2}
$$

we see the $\mathcal{L}_{H}$ spectrum as being infinitely degenerate with degeneracy label $\nu$ and

$$
2 \nu \geq \mu \geq-2 \nu
$$

When we consider analytic continuation we may keep $\nu$ fixed and continue in $\mu$; or make both $\nu$ and $\mu$ complex.

If $\left\{\psi_{z}\right\}$ is the spectral family of an operator, say the Hamiltonian $H$ and $\left\{\tilde{\psi}^{z}\right\}$ the dual family, then a basis for the space of linear functionals $\mathcal{L}$ is given by the two index family $\left\{\psi_{z} \tilde{\psi}^{z^{\prime}}\right\}$. Similarly if $\left\{\phi_{z}\right\}$ is the spectral family of $H_{0}$ and $\left\{\tilde{\phi}^{z}\right\}$ the dual family another two index family is $\left\{\phi_{z} \tilde{\phi}^{z^{\prime}}\right\}$.

It is convenient to employ the Dirac notation for the state vectors and density operators

$$
\begin{aligned}
\phi_{z} & \rightarrow|z\rangle, \tilde{\phi}^{z^{\prime}} \rightarrow\left\langle\tilde{z}^{\prime}\right| \\
\psi_{z} \rightarrow \mid z \gg, \tilde{\psi}^{z^{\prime}} \rightarrow & \ll \tilde{z}^{\prime} \mid .
\end{aligned}
$$

Then

$$
\psi_{z} \tilde{\psi}^{z^{\prime}} \rightarrow\left|z \gg \ll \tilde{z}^{\prime}\right|, \quad \phi_{2} \tilde{\phi}^{z^{\prime}} \rightarrow|z\rangle\left\langle z^{\prime}\right|
$$

We compute

$$
\begin{aligned}
i \frac{d}{d t}|z\rangle\left\langle\tilde{z}^{\prime}\right| & =H|z\rangle\left\langle\tilde{z}^{\prime}|-| z\right\rangle\left\langle\tilde{z}^{\prime}\right| H \\
& =\mathcal{L}_{H}|z\rangle\left\langle\tilde{z}^{\prime}\left|=\left(\mathcal{L}_{H_{0}}+\mathcal{L}_{V}\right)\right| z\right\rangle\left\langle\tilde{z}^{\prime}\right|
\end{aligned}
$$


It is also possible to deduce

$$
i \frac{d}{d t}\left|z \gg \ll \tilde{z}^{\prime}\right|=\mathcal{L}_{H}\left|z \gg \ll \tilde{z}^{\prime}\right|=\left(z-z^{\prime}\right)\left|z \gg \ll \tilde{z}^{\prime}\right| .
$$

In particular, since

$$
\mathcal{L}_{H}|z \gg \ll \tilde{z}|=0
$$

the quantities $|z \gg \ll \tilde{z}|$ are constants of motion.

The bilinear in the transition amplitude (generalized transition probability) is given by

$$
\begin{aligned}
\operatorname{tr}\left(\left|z \gg \ll \tilde{z}^{\prime}\right| \mathcal{L}_{H}|\zeta\rangle\left\langle\tilde{\zeta}^{\prime}\right|\right) & \left.=\left(z-z^{\prime}\right) \operatorname{tr}\left(\left|z \gg \ll \tilde{z}^{\prime}\right| \zeta\right\rangle\left\langle\zeta^{\prime}\right|\right) \\
& =\left(z-z^{\prime}\right) \ll \tilde{z}^{\prime}|\zeta\rangle\left\langle\zeta^{\prime}\right| z \gg
\end{aligned}
$$

Since

$$
\left.\left|z \gg=\left(1-G_{0+}(z) V\right)^{-1}\right| z\right\rangle
$$

the transition rate can be equally well written

$$
\left.\left(z-z^{\prime}\right)<\tilde{z^{\prime}}\left|\left(1-V G_{0+}\left(z^{\prime}\right)\right)^{-1}\right| \zeta\right\rangle\left\langle\tilde{\zeta}^{\prime}\left|\left(1-\tilde{G_{0+}}(z)\right)^{-1}\right| z\right\rangle .
$$

In particular this vanishes for $z=z^{\prime}$.

But if we attempt to calculate

$$
\operatorname{tr}\left(|z \gg \ll \tilde{z}| \mathcal{L}_{H}|\zeta\rangle\langle\tilde{\zeta}|\right)=\frac{d}{d t} \mid\left\langle\zeta|z \gg|^{2}\right.
$$

we encounter an undefined quantity. On the one hand since $\mathcal{L}_{H}$ annihilates $|z \gg \ll \tilde{z}|$ this is zero. But

$$
\mathcal{L}_{h}=\mathcal{L}_{H_{0}}+\mathcal{L}_{V}
$$

and $\mathcal{L}_{H_{0}}$ annihilates $|\zeta\rangle\langle\tilde{\zeta}|$ it appears that the time derivative is

$$
|\langle\tilde{\zeta}|V| z\rangle|^{2}
$$


which is nonzero. This ambiguity is resolved when we understand that

$$
\operatorname{tr}(|z \gg \ll \tilde{z}|,|\zeta\rangle\langle\tilde{\zeta}|)=\mid\left\langle\zeta|z \gg|^{2}\right.
$$

is undefined since it contains the square of the delta function in

$$
\langle\zeta| z \gg=\delta(\zeta-z)+\left\langle\zeta G_{0+}(z) T(z) \mid z\right\rangle
$$

Thus we should not attempt to calculate the double diagonal matrix element!

A "diagonal" initial state $|\zeta\rangle\langle\tilde{\zeta}|$ will lead to a correlated final state which is also pure and must therefore contain correlations $\left|z \gg \ll \tilde{z}^{\prime}\right|$ with $z \neq z^{\prime}$. If these are neglected we would, like Boltzmann's "proof" of the $H$-theorem, arrive at a fictitious time asymmetry, and would appear to cause time evolutions which take us from a pure state to a mixed state. Even if the initial state is a weighted sum $\int W(\zeta)|\zeta\rangle\langle\tilde{\zeta}| d \zeta$ there are correlations $\left|z \gg \ll \tilde{z}^{\prime}\right|$ in the final state for $z \neq z^{\prime}$ which must not be neglected unless there are external effects which wipe out such correlations.

A more careful calculation involving due attention to limits is the following:

$$
\begin{aligned}
i\left(\zeta-\zeta^{\prime}\right) & \ll \tilde{z}^{\prime}|\zeta\rangle\left\langle\tilde{\zeta}^{\prime}\left|z \gg+i \ll z^{\prime}\right| V \mid \zeta\right\rangle\left\langle\tilde{\zeta}^{\prime}\left|z \gg-i \ll z^{\prime}\right| \zeta\right\rangle\left\langle\tilde{\zeta}^{\prime}|V| z \gg\right. \\
& =\operatorname{tr}\left(\left|z \gg \ll \tilde{z}^{\prime}\right|\left(\mathcal{L}_{H_{0}}+\mathcal{L}_{V}\right)|\zeta\rangle\left\langle\tilde{\zeta}^{\prime}\right|\right) .
\end{aligned}
$$

We know that

$$
\left\langle\tilde{\zeta}^{\prime}\right| z \gg=\left\langle\tilde{\zeta}^{\prime} \mid z\right\rangle+\int T(\nu, z) \frac{d \nu}{z-\nu+i \eta}\left\langle\tilde{\zeta}^{\prime} \mid z\right\rangle
$$

and that

$$
\begin{aligned}
\left(\zeta^{\prime}-z\right)\left\langle\tilde{\zeta}^{\prime}\right| z \gg & =-T\left(\zeta^{\prime}, z\right) \\
\left(z^{\prime}-\zeta\right) \ll \tilde{z}^{\prime}|\zeta\rangle & =-\tilde{T}\left(\zeta, z^{\prime}\right)
\end{aligned}
$$

Hence

$$
i\left(\zeta^{\prime}-\zeta\right)\left\langle\tilde{\zeta}^{\prime}\left|z \gg \ll \tilde{z}^{\prime}\right| \zeta\right\rangle=-\left\{T\left(\zeta^{\prime}, z\right) \ll \tilde{z}^{\prime}|\zeta\rangle+\tilde{T}\left(\zeta, z^{\prime}\right)\left\langle\tilde{\zeta}^{\prime}\right| z \gg ;\right.
$$




\section{•}

and

$$
\begin{aligned}
\left.i \ll z^{\prime}|V| \zeta\right\rangle\left\langle\tilde{\zeta}^{\prime}\right| z \gg & =i \tilde{T}\left(\zeta, z^{\prime}\right)\left\langle\tilde{\zeta}^{\prime}\right| z \gg \\
-i \ll z^{\prime}|\zeta\rangle\left\langle\tilde{\zeta}^{\prime}|V| z \gg\right. & =-i T\left(\zeta^{\prime}, z\right) \ll z^{\prime}|\zeta\rangle
\end{aligned}
$$

Hence

$$
\operatorname{tr}\left(\left|z \gg \ll \tilde{z}^{\prime}\right|\left(\mathcal{L}_{H_{0}}+\mathcal{L}_{V}\right)|\zeta\rangle\left\langle\tilde{\zeta}^{\prime}\right|=\left(z-z^{\prime}\right) \operatorname{tr}\left(\left|z \gg \ll \tilde{z}^{\prime}\right| \zeta\right\rangle\left\langle\tilde{\zeta}^{\prime}\right|\right)
$$

which does not vanish for $\zeta^{\prime}=\zeta$ for $\cdot z^{\prime} \neq z$. The right hand side is exactly

$$
\begin{aligned}
\operatorname{tr}\left\{\left(\mathcal{L}_{H}\left|z \gg \ll \tilde{z}^{\prime}\right|\right)|\zeta\rangle\left\langle\zeta^{\prime}\right|\right\} & \left.=i \frac{d}{d t}\left(\left|z, t \gg \ll \tilde{z}^{\prime}, t\right| \zeta\right\rangle\langle\zeta|\right) \\
& =i \frac{d}{d t}\left(\left\langle\tilde{\zeta}^{\prime}\left|z, t \gg \ll z^{\prime}, t\right| \mid \zeta\right\rangle\right) .
\end{aligned}
$$

It would therefore be vanishing for $z^{\prime}=z$ and $\int|z, t \gg \ll \tilde{z, t}| Y(z) d z$ are stationary density operators of the system. There are arbitrarily many stationary density operators, one for each function $Y(z)$.

\section{Concluding Remarks}

In this paper we have shown that the natural formalism for quantum mechanics is in terms of families of vector spaces of analytic functions and their duals. The various choices of the generalized spaces $\mathcal{G}$ give the same physical quantities in terms of differenc contour intergrals. One particular use is to study resonances in scattering systems. The method developed in thic paper can also compute the scattering of a resonance off a particle. Scattering theory is reformaulated using a comparison Hamiltonian along with the total Hamiltonian. This comparison Hamiltonian takes account of the change of the spectrum due to interaction and is an essential element of setting up a proper scattering theory. In addition to the change in the spectrum, corresponding to mass renormalization in quantum field theory there is also a possible wavefunction normalization. Examples of both are worked out in models of interacting field theories. A perturbation 
theory for interacting systems with both continuous and discrete spectra is formulated. It is shown how the perturbation series can be formally summed to give closed form expressions for the Möller matrix.

It is important in this context to note that the constructive definition of the Möller matrix shows how to get the modified spectrum and the generalized Möller matrix. The failure of the usual adiabatic hypothesis and the simpleminded asymptotic condition is seen even in these simple models. When the correct asymptotic condition is invoked and the properly generalized Möller matrix is evaluated, the latter is not only isometric but unitary; and acts as an intertwining operator between the total Hamiltonian and the comparison Hamiltonian. The unreliability of the singularities of the scattering amplitude as an indicator of the physical spectrum is also brought out.

The perturbation theory is equally adapted for the analytic continuation to the spaces $\mathcal{G}$. The resonant states can be identifies as discrete states in $\mathcal{G}$. The entire formalism of "in" and "out" states and the generalized unitary Möller matrices can be applied equally well to the analytic continuations. So when a discrete "particle" state becomes unstable by perturbation, in suitable analytic continuation, we can continue to use perturbation theory. This method can also be used for determining resonance energies in interacting systems.

The question of temporal evolution of density matrices can be handled in terms of the Liouville operator and its exponentation. The analytic continuation of the second vector space obtained as the span of the density matrices is examined and the question of time symmetry is also investigated. We can again find semigroups of time evolutions; but the original density matrices continue to admit of the full group of time evolutions.

The present formalism of dual spaces is different from the rigged Hilbertspace theory which also deals with dual spaces. But the dual spaces are the primary entries here. Some earlier papers in the literature claiming time asymmetry obtain their results by introducing unphysical states with energies unbounded from below. While such "states" can be constructed from physical 
states by Hilbert trasforms they are a caricature of the physical state. Similar constructions with density matrices are also outlined and criticized.

The present work is in one sense the completion of the Heisenberg program to make dynamics out of directly measured quantities like spectral frequencies and intensties augments by resonance positions and widths; and in another sense a minor generalization of the Dirac formalism of quantum theory in terms of ket and bra vectors. It is instructive that these old ideas contain the germs of many modern developments.

The author has benefitted from disucssions with Gopalakrishnan Bhamathi, Ilya Prigogine and Ioannis Antoniou and for their encouragement. This work is supported by the Department of Energy Contract ER40757-020. 


\section{References}

[1] PAM Dirac, "Principles of Quantum Mechanics", Oxford University Press (4th edition (Oxford 1954)).

[2] J. von Neumann, "Mathematical Foundations of Quantum Mechanics", Springer-Verlag (Berlin 1932).

[3] E.C.G. Sudarshan, C.B. Chiu and V. Corini, Phys. Rev D18, 2914 (1978).

[4] E.C.G. Sudarshan, Phys. Rev A46, 37 (1992).

[5] A. Bohm, J. Math. Phys. 21, 1040 (1980); 22, 2313 (1981); A. Bohm and M. Gadella, "Dirac Kets, Gamow Vectors and Gelfand Triplets", Springer Lecture Notes in Physics Vol. 348 Springer-Verlag, (Berlin, 1989).

[6] E.C.G. Sudarshan and Charles B. Chiu, Phys. Rev. D47, 2602 (1993).

[7] J.G. Kuriyan, N. Mukunda and E.C.G. Sudarshan, Comm. Math. Phys. 8, 204 (1968); J. Math. Phys. 9, 12 (1968);

G. Lindbladt, Comm. Math. Phys. 48, 119 (1976).

[8] H. Weyl, Classical Groups, Princeton University Press, (Princeton 1939). P.A.M. Dirac, Proc. Roy. Soc. A287, 532 (1965).

Harish-Chandra, Proc. Roy. Soc. A189, 372 (1947).

[9] T.D. Lee, Phys. Rev. 95, 1329 (1954);

K.O. Friedrichs, Comm. Pure and Appli. Math. 1, 361 (1948);

P.A.M. Dirac, ref 1. p. $206 \mathrm{Eq}(52)$.

[10] E.C.G. Sudarshan, "Relativistic Particle Interaction", Notes by V. Teplitz, Proc. of the 1961 Brandies Summer Institute, Benjamin, (New York 1962). 
[11] I. Antoniou and S. Tasaki, Journal of Physics A26, 73 (1993).

[12] E.C.G. Sudarshan, Charles B. Chiu and G. Bhamathi, Phys. Rev. D46, 3508 (1992).

[13] E.C.G. Sudarshan, Charles B. Chiu and G. Bhamathi, sumitted to Physica ...

[14] S.T. Ma, Phys. Rev. 69, 668 (1946); 71, 195 (1947);

S.N. Bisqas, T. Pradham and E.C.G. Sudarshan, Nucl. Phys. B50, 269 (1972).

[15] I. E. Segal, Annals of Math. 48, 930 (1947).

[16] T. Petrosky and I. Progogine, Proceedings of Nat. Acad. Sc. (US) (in press).

[17] B. Misra and E.C.G. Sudarshan, J. Math. Phys. 18756 (1977).

[18] E.C.G. Sudarshan, Charles B. Chiu and B. Misra, phys. Rev. D16, 520 (1977).

[19] C. Möller, K. Dansk, Vid. Selsk. Mat-Fys. Medd. 23, 1 (1945);

J.M. Jauch, Helvetica Physica Acta 31, 127 (1958); 31, 661 (1958).

[20] G. Breit and E.P. Wigner, Phys. Rev. 57, 472; 642 (1936).

[21] J. Math. Phys. 21, 1040 (1980); 22, 2313 (1981); Physica 124A, 103 (1984).

[22] T.F. Jordan, A.J. Macfarlane and E.C.G. Sudarshan, Phys. Rev. 132, 487 (1964).

[23] E.P. Wigner and J. von Neumann, Z. Phys. 30, 465 (1929;

B. Simon, Comm. Pure Appl. Math. 22, 531 (1967); E.C.G. Sudarshan, in "Field Theory, Quantizaiton and Statistical Physics" ed. E. Terapegui (Reidel, Dodrecht 1981.

[24] Y. Yamaguchi, Phys. Rev. 95, 1628 (1954); Progr. Theor. Phys. Suppl. 7, 1 (1958);

Yoshio Yamaguchi and Yoriko Yamaguchi, Phys. Rev. 95, 1635 (1954). 


\section{Ërata}

p. 6, equation (3) should read:

$$
\begin{aligned}
(\Pi(\{\mu\}) \phi)(\{\lambda\}) & =\theta(\{\lambda\},\{\mu\}) \phi(\{\mu\}) . \\
\theta(\{\lambda\},\{\mu\}) & =\left\{\begin{array}{ll}
1 & \{\lambda\} \geq\{\mu\} \\
0 & \text { otherwise }
\end{array},\right.
\end{aligned}
$$

p. 12, equation (32) should read: $\quad \rho(t)=\sum_{n} a_{n} \int_{R^{+}} d \lambda\left\{\int_{-2 \lambda}^{2 \lambda} \psi_{n}\left(\lambda+\frac{\nu}{2}\right) \phi_{n}^{*}\left(\lambda-\frac{\nu}{2}\right) e^{-i \nu t} d \nu\right\}$

$$
=\sum_{n} a_{n} \int_{-\infty}^{\infty} e^{-i \nu t}\left\{\int_{|\nu| / 2}^{\infty} \psi_{n}\left(\lambda+\frac{\nu}{2}\right) \phi_{n}^{*}\left(\lambda-\frac{\nu}{2}\right) d \lambda\right\} d \nu .
$$

p. 14, equation (39) should read:

$$
\begin{array}{r}
\psi_{n}\left(\zeta+\frac{z}{2}\right) \psi_{m}^{*}\left(\zeta^{*}-\frac{z^{*}}{2}\right) \rightarrow \psi_{n}\left(\zeta+\frac{z}{2}\right) \psi_{m}^{*}\left(\zeta^{*}-\frac{z^{*}}{2}\right) e^{-i z t} \\
i \frac{d}{d t}\left\{\psi_{n}\left(\zeta+\frac{z}{2} t\right) \psi_{m}^{*}\left(\zeta^{*}-\frac{z^{*}}{2}, t\right)\right\}=z\left\{\psi_{n}\left(\zeta+\frac{z}{2}, t\right) \psi_{m}^{*}\left(\zeta^{*}-\frac{z^{*}}{2} t\right)\right\} .
\end{array}
$$

p. 16, equation (51) should read:

$$
\Psi_{\lambda}=\Psi_{o}+\int\left(\Psi_{o \omega}^{+} T(\lambda) \Psi_{o \lambda}\right) G_{o}(\lambda) \Psi_{o \omega} d \omega
$$

p. 20, equation (60) should read:

$$
\begin{aligned}
|\psi\rangle & =\left|\phi_{+}\right\rangle+\left|\phi_{-}\right\rangle \\
\left|\phi_{ \pm}\right\rangle & =\frac{ \pm 1}{2 \pi i} \int_{\Gamma^{\prime}}\left\{\int_{\Gamma} \frac{a\left(z^{\prime}\right)}{z-z^{\prime} \pm i \eta} d z^{\prime}+\frac{a_{n}}{z-z_{n} \pm i \eta}\right\}|z\rangle d z
\end{aligned}
$$

p. 24, equation (78) should read:

$$
\begin{aligned}
\rho(t) & =\rho_{+}(t)+\rho_{-}(t) \\
\rho_{ \pm}(t) & =\int_{0}^{\infty} d \lambda \int_{-\infty}^{\infty}\left|\lambda+\frac{\mu}{2} \gg \ll \lambda-\frac{\mu}{2}\right| e^{-i \mu t}\left\{\int_{-\frac{\lambda}{2}}^{\frac{\lambda}{2}} \frac{ \pm 1}{2 \pi i} \rho\left(\lambda ; \mu^{\prime}\right) \frac{1}{\mu-\mu^{\prime} \pm i \epsilon} d \mu^{\prime}\right\} d \mu .
\end{aligned}
$$

p. 38, the first displayed but unnumbered equation should read:

$$
\begin{aligned}
\Omega^{+} \Omega & =1, \quad \Omega \Omega^{+}=1-\mathcal{P}_{0} \\
\Omega_{1} \Omega_{1}^{+}|z\rangle & =Z|z\rangle=\sum_{m, n=0}^{\infty}\left(V G^{+}(z)\right)^{m} \cdot(G(z) V)^{n}|z\rangle
\end{aligned}
$$



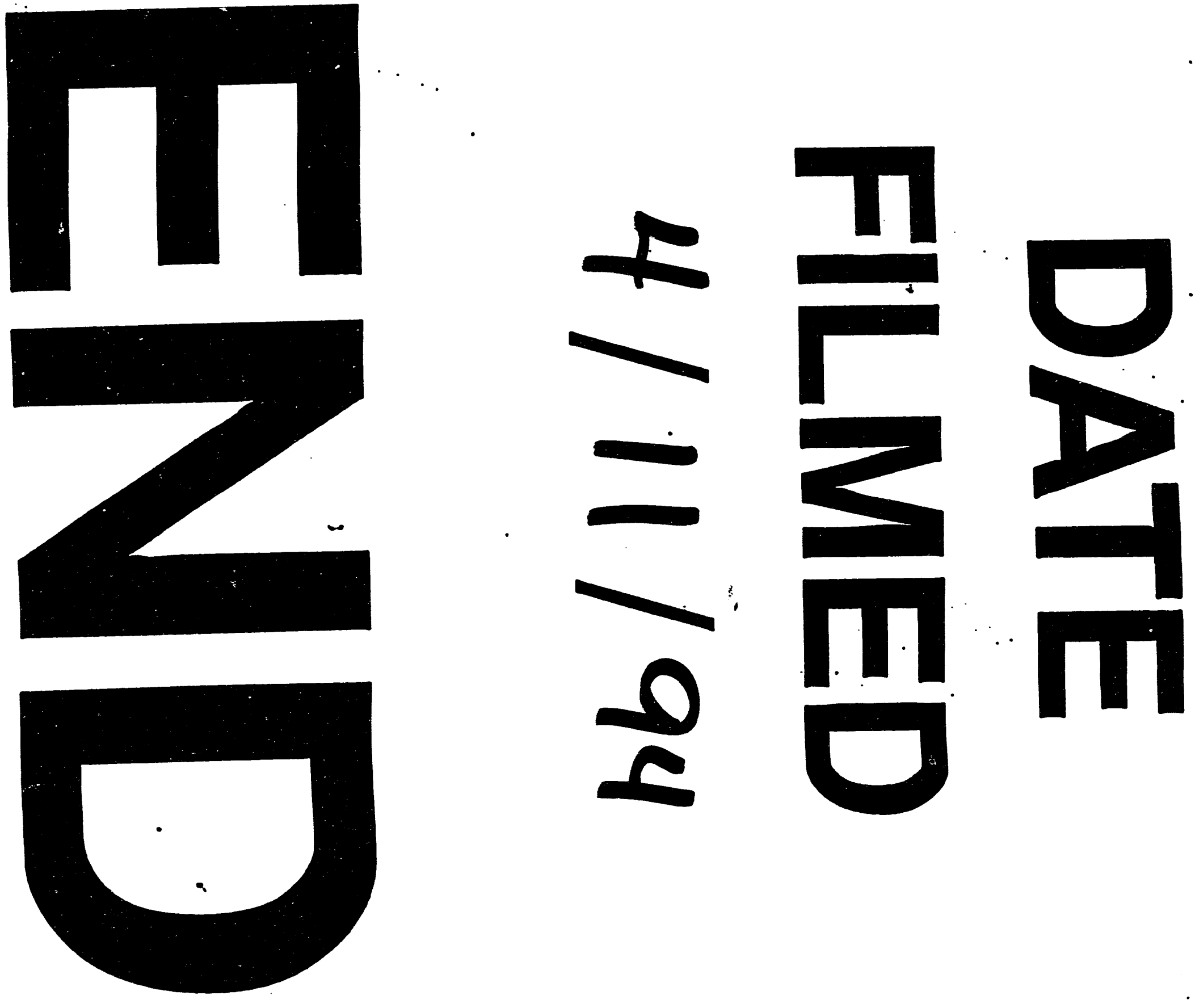

$-$

.

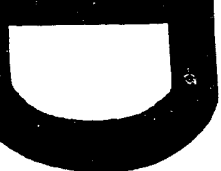


